\title{
OBSERVATIONS OF THE OCULAR GALAXY NGC 2535 AND ITS STARBURST COMPANION
} NGC 2536

\author{
MiCHELE KAUFMAN \\ Department of Physics and Department of Astronomy, Ohio State University, 174 W. 18th Avenue, Columbus, Ohio 43210 \\ Electronic mail: rallis@mps.ohio-state.edu \\ ELIAS BRINKS \\ Depto. de Astronomía, Universidad de Guanajuato, Apdo. Postal 144, Guanajuato, Gto. 36000, México \\ Electronic mail: ebrinks@coyotl.astro.ugto.MX \\ DEBRA MELOY ELMEGREEN \\ Vassar College Observatory, Poughkeepsie, New York 12601 \\ Electronic mail: elmegreen@vaxsar.vassar.edu \\ MAGNUS THOMASSON \\ Onsala Space Observatory, S-439 92 Onsala, Sweden \\ Electronic mail: magnus@oso.chalmers.SE \\ BRUCE G. ELMEGREEN \\ IBM Research Division, T. J. Watson Research Center, P.O. Box 218, Yorktown Heights, New York 10598 \\ Electronic mail: bge@watson.ibm.com
}

CURTIS STRUCK

Department of Physics and Astronomy, Iowa State University, Ames, Iowa 50010

Electronic mail: curt@iastate.edu

MARIO KLARIĆ

Midlands Technical College, Columbia, South Carolina 29202

Electronic mail: klaricm@mtc2.mid.tec.sc.us

Received 1997 July 9; revised 1997 August 25; accepted 1997 August 26

\begin{abstract}
$\mathrm{H}$, radio continuum, and ${ }^{12} \mathrm{CO} J=1 \rightarrow 0$ observations at resolutions of $12^{\prime \prime}$ to $33^{\prime \prime}(=2.9-8 \mathrm{kpc})$, and $B$, $I, J$, and $K$-band images, are presented of the galaxy NGC 2535 and its small starburst companion NGC 2536. NGC 2535 has an ocular (eye-shaped) structure with two long tidal arms indicative of a recent, close, nonmerging encounter. Our observations reveal widespread high velocity dispersions $\left(30 \mathrm{~km} \mathrm{~s}^{-1}\right)$ in the $\mathrm{H}$ I gas and five clouds with masses of $\sim 10^{8} \mathscr{M}_{\odot}$ in the tidal arms of NGC 2535. These clouds do not correspond to the most luminous blue knots or to major clumps in the old stellar population. Similar phenomena were found previously in other pairs (IC 2163/NGC 2207 and NGC 5774/5), suggesting that enhanced turbulence and massive gas clouds may be a general feature of interacting spiral galaxies during an early phase of post-encounter evolution. A search for ${ }^{12} \mathrm{CO} \mathrm{J}=1 \rightarrow 0$ emission at 11 positions in NGC 2535 and one position in NGC 2536 yielded two clear detections, one at the center of NGC 2535 and the other on its tidal tail but close to the center of NGC 2535. Most of the massive H I clouds in NGC 2535 are not detected in $\mathrm{CO}$ emission. The virial masses of these clouds are significantly greater than their measured masses in gas, presumably because of the presence of old disk stars in the clouds. A form of the virial theorem that includes gas and stars is presented. NGC 2535 shows several unusual features, including an intrinsically oval shape to the disk, that are similar to those in the ocular galaxy IC 2163 studied previously. NGC 2535 also contains structures that are not seen in IC 2163. For example, there is an extended ( $R$ $=48 \mathrm{kpc}) \mathrm{H} \mathrm{I}$ envelope and a long, outer, elliptically-shaped H I arc in NGC 2535 that may be a gravitational wake produced by the passage of the companion within or close to the envelope. In the radio continuum, NGC 2535 exhibits a bar-like feature that leads the small stellar bar by $50^{\circ}$. The starburst companion, NGC 2536 , lies in a $2 \times 10^{9} \mathscr{M}_{\odot}$ clump of H I gas at the outer end of the tidal bridge from NGC 2535. Most of the gas in this clump is associated with the bridge. The H I emission on the anticompanion side of NGC 2536 has the same line-of-sight velocity and projected position as some of the bridge gas there. This observation is consistent with a previous model by Klaric in which NGC 2536 accretes gas from NGC 2535; we even find an $\mathrm{H}$ I feature that may represent bridge gas streaming towards
\end{abstract}


NGC 2536. The failure to detect ${ }^{12} \mathrm{CO}$ emission in NGC 2536 places an upper limit of $6 \times 10^{8} \mathscr{M b}_{\odot}$ on its $\mathrm{H}_{2}$ mass if the standard value of the conversion factor between $I_{\mathrm{CO}}$ and $N\left(\mathrm{H}_{2}\right)$ applies. (C) 1997 American Astronomical Society. [S0004-6256(97)01412-X]

\section{INTRODUCTION}

The interacting galaxies NGC 2535 and NGC 2536 are one of five nearby galaxy pairs that we have observed with the VLA ${ }^{1}$ and other telescopes to study structures and motions indicative of recent, close, non-merging encounters. Such encounters may trigger a range of phenomena, including grand-design spirals, bars, starbursts in the disks or nuclei, warps, and the formation of dwarf galaxy companions. Observations of another member of this sample, IC 2163/ NGC 2207 (Elmegreen et al. 1995a, hereafter Paper I) revealed an intrinsically oval shape to the disk of IC 2163, widespread high velocity dispersions $\left(30-50 \mathrm{~km} \mathrm{~s}^{-1}\right)$ in the $\mathrm{H}$ I gas, a growing warp of the disk of NGC 2207, and eleven unusually massive $\left(10^{8}-10^{9} \mathscr{C}_{\odot}\right) \mathrm{H}$ I clouds not associated with bright stellar knots. These features are in good agreement with computer simulations (Elmegreen 1995b, hereafter Paper II). Elmegreen et al. (1993) suggested on the basis of these observations that close interactions increase gas turbulence and the gravitational Jeans mass of the largest clouds.

In NGC 5774, the face-on member of the interacting pair NGC 5774/75, Irwin (1994) similarly found large areas of enhanced $\mathrm{HI}$ velocity dispersion and three $\mathrm{HI}$ clouds with masses in the range $10^{7}-10^{8} \mathscr{M}_{\odot}$ and no bright stellar knots at the same location. On the other hand, in five galaxymerger pairs, Hibbard (1995) notes that the H I velocity dispersions in the tidal tails are generally low, although they are somewhat higher in the $\mathrm{H}$ I clumps; these mergers are more evolved than the non-merging pairs we are studying. Smith (1994) finds $10 \mathrm{H} \mathrm{I}$ clouds with masses of 3 $\times 10^{7}-10^{8} \mathscr{Q}_{\odot}$ and no strong star formation on the western tail of NGC 2782, an isolated galaxy whose peculiar structure appears to result from a previous collision or merger. The behavior of the H I velocity dispersion in this tail is similar to that in Hibbard's merger pairs, i.e., the velocity dispersions are slightly elevated in the clumps not in the rest of the tail [see Smith (1997a) for revised values of the velocity dispersions]. In the bridge/ring interacting pair NGC 7714/15, Smith et al. (1997) and Smith (1997b) find that the $\mathrm{H}$ I velocity dispersion is generally low except in the nucleus and close to $\mathrm{H}$ II regions.

We present $\mathrm{H} \mathrm{I}$ and radio continuum observations taken at the VLA with $\mathrm{C}$ and $\mathrm{D}$ arrays, ${ }^{12} \mathrm{CO} J=1 \rightarrow 0$ data from Onsala Space Observatory, ${ }^{2}$ optical images taken with the Burrell-Schmidt telescope at KPNO, and near-infrared images taken with the $2.1 \mathrm{~m}$ telescope at KPNO of NGC

${ }^{1}$ The National Radio Astronomy Observatory is operated by Associated Universities, Inc., under cooperative agreement with the National Science Foundation.

${ }^{2}$ Onsala Space Observatory at Chalmers University of Technology is the Swedish National Facility for Radio Astronomy.
2535/36 (Arp 82). NGC 2535 has an eye-shaped (“ocular") structure consisting of a sharply pointed oval with two long tidal arms [see Fig. 1 (Plate 101), which displays a skysubtracted $B$-band image of NGC 2535 and its companion NGC 2536]. In optical morphology, NGC 2535 resembles the ocular galaxy IC 2163 studied in Paper I. Numerical simulations of close, prograde, approximately in-plane encounters between galaxies indicate that such structures develop as a short-lived phase one-half revolution after perigalacticon (see, for example, Elmegreen et al. 1991 and Sundin 1993). The advantages of studying ocular galaxies are (1) the geometry and time of the interaction are well constrained by the morphology and (2) they show many features typical of a strong tidal response without the complication of stellar population mixing that arises in a direct collision or merger. In the $\mathrm{H} \alpha$ Fabry-Perot observations by Amram et al. (1989) at $1.5^{\prime \prime}$ resolution, NGC 2535 exhibits a nearly constant velocity along the tidal arms. Since their observations predated much of the theoretical work on the velocity field of oculars, Amram et al. did not recognize this velocity anomaly as an expected characteristic resulting from streaming motions.

The companion, NGC 2536, is a small bright galaxy at the end of the long tidal arm of the larger galaxy, NGC 2535. Arp (1969) points out a very bright knot of [O III] emission 5 " NE of the nucleus of NGC 2536. From optical spectroscopy, Bernlöhr (1993) concludes that NGC 2536 is undergoing a burst of star formation, with a burst age of $\sim 10^{7}$ years, whereas he lists NGC 2535 as having somewhat enhanced star formation but not a starburst. Bernlöhr classifies the emission-lines in both galaxies as $\mathrm{H}$ II region type, i.e., not Seyfert or LINER.

Galaxy encounter simulations that match the optical morphology of NGC 2535 have a companion-to-ocular mass ratio of 0.1 in a 2D model by Howard et al. (1993) and 0.3 in a 3D model by Klarić (1993). In Klarić's model, NGC 2536 approached NGC 2535 from the northwest and above the disk, crossed the plane of the disk north of the nucleus, and then traveled underneath NGC 2535 until recently; it is now crossing the disk of NGC 2535 from the far side to the near side. This trajectory, which has an initial tilt of $30^{\circ}$ with respect to the plane of NGC 2535, is drawn in Fig. 2. Perigalacticon occurred $\sim 10^{8}$ years ago at a distance of $12.4 \mathrm{kpc}$ (just outside of the initial simulated disk of NGC 2535). The maximum $z$ displacement of NGC 2536 from the plane of NGC 2535 between disk crossings was $\sim 6 \mathrm{kpc}$. In Klarić's model, NGC 2536 accretes gas from NGC 2535, and this may have fueled the starburst in NGC 2536. His model did not describe the velocity fields, so additional simulations (to be published later) are in progress.

Throughout this paper, we will be comparing the tidal features in NGC 2535/36 with those in IC 2163/NGC 2207. NGC 2535 and IC 2163 both exhibit ocular structure, but 

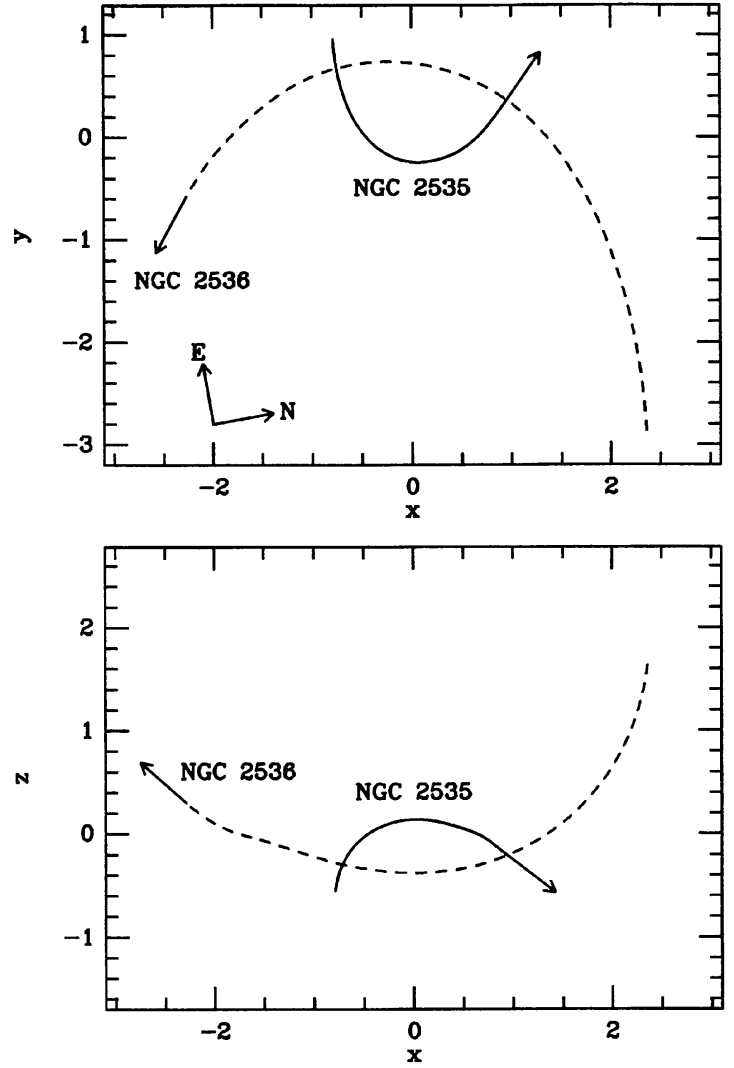

FIG. 2. The orbits of NGC 2535 (solid curve) and NGC 2536 (dashed curve) in the center-of-mass frame from Klaric (1993). The $x y$ plane is the plane of the initial disk of NGC 2535, and the $z$ direction is positive towards us. The arrows at the ends of the curves indicate the directions of motion of the galaxies. The length unit in these diagrams corresponds to $11.4 \mathrm{kpc}$.

they differ in ocular/companion mass ratio. NGC 2535 is a large ocular with a small companion, whereas IC 2163 is an ocular with a mass nearly equal to the companion's. Also, according to the encounter simulations (Klarić's model for NGC 2535/36 and Paper II for IC 2163/NGC 2207), more time has elapsed since perigalacticon in the NGC 2535/36 pair (100 Myr) than in the IC 2163/NGC 2207 pair (40 Myr), and the tilt of the companion's orbit relative to the plane of the ocular is slightly greater for NGC 2535 than for IC 2163. There is presently a starburst in NGC 2536 but not in any of the larger galaxies NGC 2207, IC 2163, and NGC 2535. Nevertheless, both IC 2163 and NGC 2535 have bright knots of star formation on the eye-shaped ovals. Other similar features will be demonstrated below: widespread, enhanced turbulence in the $\mathrm{H}$ I gas, intrinsically oval shapes to the disks, unusually massive $\mathrm{H}$ I clouds without major stellar clumps, and a concentration of most of the outer disk stars into the tidal arms.

The distance to NGC $2535 / 36$ is assumed to be $50 \mathrm{Mpc}$, based on $H_{0}=80 \mathrm{~km} \mathrm{~s}^{-1} \mathrm{Mpc}^{-1}$ from Freedman et al. (1994); then $1^{\prime \prime}=240 \mathrm{pc}$. Table 1 lists the basic galaxy properties from the Third Reference Catalogue (de Vaucouleurs
TABLE 1. Basic data on NGC 2535/36.

\begin{tabular}{|c|c|c|}
\hline CHARACTERISTIC & NGC 2535 & NGC 2536 \\
\hline Morphological Type & SA(r)c pec & SB(rs)c pec \\
\hline Right Ascension (1950) & $08^{h} 08^{m} 13.12^{a}$ & $08^{h} 08^{m} 15.63^{\prime}$ \\
\hline Declination (1950) & $+25^{\circ} 21^{\prime} 21.8^{\prime \prime}$ & $+25^{\circ} 19^{\prime} 43.4^{\prime \prime}$ \\
\hline Isophotal major radius $R_{25}$ & $1.25^{\prime}$ & $0.45^{\prime}$ \\
\hline Axis ratio & 2.1 & 1.5 \\
\hline$B$ magnitude $B_{T}$ & 13.31 & 14.70 \\
\hline Corrected $\mathrm{B}_{T}^{0}$ & 12.64 & 14.32 \\
\hline$(B-V)_{T}(\operatorname{mag})$ & 0.54 & 0.55 \\
\hline Optical heliocentric $\mathrm{v}\left(\mathrm{km} \mathrm{s}^{-1}\right)$ & 4095 & 4085 \\
\hline Galactic $A_{B}$ (mag) & \multicolumn{2}{|c|}{0.14} \\
\hline $\mathrm{f}(60 \mu \mathrm{m})(\mathrm{J} y)$ & \multicolumn{2}{|c|}{2.24} \\
\hline $\mathrm{f}(100 \mu \mathrm{m})(\mathrm{Jy})$ & \multicolumn{2}{|c|}{6.12} \\
\hline Distance (Mpc) & \multicolumn{2}{|c|}{50} \\
\hline
\end{tabular}

et al. 1991) and the NASA Extragalactic Database (NED). ${ }^{3}$ The velocities are taken from Amram et al. (1989) and the values of the right ascension, declination, and distance, are from this paper. All of the velocities given in this paper are heliocentric, optical definition.

Section 2 describes the observations and the data reduction. The properties of NGC 2535/36 in the optical and nearinfrared, in ${ }^{12} \mathrm{CO} J=1 \rightarrow 0$ emission, in $\mathrm{H}$ I emission, and in radio continuum emission, are presented in Secs. 3, 4, 5, and 6 , respectively. Optical surface photometry of NGC 2535 is described in Sec. 7. In Sec. 8, we consider whether the outer elliptical $\mathrm{H}$ I arc found in our images could be a gravitational wake. Section 9 discusses the results, presents a table (Table 6) comparing the disturbances in the ocular galaxies NGC 2535 and IC 2163, and summarizes our conclusions.

\section{OBSERVATIONS AND DATA REDUCTION}

\subsection{Optical and Near Infrared}

Images of NGC $2535 / 36$ in $B$ and $I$ bands were taken with the Burrell-Schmidt telescope using the $s 2 k a$ CCD detector at Kitt Peak. The images were bias subtracted, flat fielded, combined, and sky subtracted using standard IRAF procedures. The $I$-band image, a $1053 \mathrm{~s}$ exposure taken on 1993 January 29, has a PSF of $5.0^{\prime \prime} \times 5.9^{\prime \prime}$. The $B$-band image combines a $1000 \mathrm{~s}$ exposure taken on 1993 January 29 with $\mathrm{PSF}=4.2^{\prime \prime} \times 3.6^{\prime \prime}$ and a $1122 \mathrm{~s}$ exposure taken on 1995 April 1 with $\mathrm{PSF}=5.3^{\prime \prime} \times 5.2^{\prime \prime}$. The final images have a pixel size of $1.47^{\prime \prime}$. From the raw images, we selected a field of view $\left(18^{\prime} \times 17^{\prime}\right.$ for the 1993 January images) that included NGC $2535 / 36$ plus 12 standard stars in the Guide Star Catalog. Using the positions of these 12 standard stars, we transformed the CCD images to right ascension and declination coordinates; the plate solutions have standard deviations $\leqslant 0.3^{\prime \prime}$. This allowed us at a later stage to bring CCD and radio images to the same coordinate grid.

We took $J$ - and $K$-band images of the central portions of NGC 2535 and NGC 2536 with the $2.1 \mathrm{~m}$ telescope and the COB detector at Kitt Peak on 1996 April 31-May 5. The images were sky subtracted and flat fielded with a global flat image made from the skies of the whole observing run. The

\footnotetext{
${ }^{3}$ The NASA/IPAC Extragalactic Database (NED) is operated by the Jet Propulsion Laboratory, Caltech, under contract with the National Aeronautics and Space Administration.
} 
exposures alternated galaxy and off-galaxy images with equal exposure times so that local skies could be made. The combined images for NGC 2535 include five $J$-band images with a total exposure time of $1380 \mathrm{~s}$ and five $K$-band images with a total exposure of $900 \mathrm{~s}$ and have a total field of view of approximately $60^{\prime \prime} \times 60^{\prime \prime}$. There is an E-W elongation caused by a telescope oscillation. The combined images for NGC 2536 include three $J$-band images with a total exposure time of $900 \mathrm{~s}$ and three $K$-band images with a total exposure time of $540 \mathrm{~s}$ and have a total field of view of approximately $50^{\prime \prime} \times 50^{\prime \prime}$. The scale is $0.2^{\prime \prime}$ per pixel, and the average seeing was $1^{\prime \prime}$.

\section{$2.2 \mathrm{CO}$}

Single-dish ${ }^{12} \mathrm{CO} J=1 \rightarrow 0$ observations were made with the $20 \mathrm{~m}$ telescope at Onsala Space Observatory on four occasions: 1994 April 9-14, 1994 May 21-22, 1995 January 10-18, and 1995 March 28-April 3. The telescope was equipped with a cooled SIS receiver. The back end consisted of a filterbank with 512 channels and a frequency resolution of $1 \mathrm{MHz}$ (corresponding to a velocity resolution of $2.6 \mathrm{~km} \mathrm{~s}^{-1}$ at $115 \mathrm{GHz}$ ). The beamsize (HPBW) was 33", and the main beam efficiency 0.5 . We used the beamswitch observing method with a beam throw of $12^{\prime}$. The standard chopper wheel method was used for calibration. The system temperature (including atmosphere) was typically between 500 and $700 \mathrm{~K}$, occasionally somewhat larger. Pointing was checked regularly using $\mathrm{SiO}$ masers (e.g., R Cas, IK Tau, V Cam, TX Cam, and R Leo) as reference sources. The pointing errors were estimated to be less than one tenth of the beamsize. We observed 11 positions in NGC 2535 and 1 position in NGC 2536. The average integration time per position was $2.2 \mathrm{~h}$, ranging from 0.8 to $3.4 \mathrm{~h}$, giving a total of $26.5 \mathrm{~h}$ (all numbers given as time on-source). The data were reduced using Data Reduction Package (DRP, developed by Michael Olberg at Onsala Space Observatory). The subtracted baselines were in almost all cases of first or second order (in the latter case still very close to straight lines). The intensities from the chopper wheel calibration, given on the $T_{A}^{*}$ temperature scale, were divided by the main beam efficiency $\left(\eta_{\mathrm{mb}}=0.5\right)$ to get the main beam temperatures $T_{\mathrm{mb}}$. The total spectra were Hanning filtered and then smoothed to a velocity resolution of $5.2 \mathrm{~km} \mathrm{~s}^{-1}$, resulting in noise with a typical $\mathrm{rms}$ value of $0.014 \mathrm{~K}$ (on the $T_{\mathrm{mb}}$ temperature scale).

\section{$2.3 H I$}

We observed NGC 2535/36 in $\mathrm{H}$ I at the VLA for 4 hours (on the target) in C configuration on 1993 July 11 and for 1 hour in D configuration on 1993 October 30. In both cases, $0738+313$ served as the phase calibrator, and $3 \mathrm{C} 286$, as the flux standard and bandpass calibrator. We assumed a flux density of $14.9 \mathrm{Jy}$ for 3C 286 on the Baars et al. (1977) scale. The data were taken in the 4IF correlator mode with two passbands, each $1.56 \mathrm{MHz}$ wide and containing 64 channels, and with a 16-channel overlap between the two bands. This covers a combined range of $594 \mathrm{~km} \mathrm{~s}^{-1}$ with a channel width of $24.414 \mathrm{kHz}=5.3 \mathrm{~km} \mathrm{~s}^{-1}$ after on-line Hanning smoothing. H I emission is present for heliocentric velocities $3972-4205 \mathrm{~km} \mathrm{~s}^{-1}$; the line-free channels were averaged to create continuum images.

The AIPS software package was used for the data reduction. The uv data from each of the VLA configurations and each of the two IFs were calibrated separately, and the stronger continuum sources were subtracted from the uv data. For each uv data set separately, we generated "dirty" data cubes of line plus residual continuum emission and subtracted the residual continuum emission from each channel. The resulting $\mathrm{H}$ I maps were cleaned and inspected. The process was successful in removing the solar interference present in part of the original $\mathrm{C}$-configuration data. Then the uv data sets from $\mathrm{C}$ and D configurations (after removal of the strong continuum sources) were combined, the above mapping and cleaning procedure repeated, first with natural weighting and then with uniform weighting, and the cubes from the two IFs were appropriately merged. We selected the areas of genuine $\mathrm{H}$ I emission by the following procedure. Since this system has rather extended $\mathrm{HI}$ emission, the naturally-weighted cube was convolved to $60^{\prime \prime}$ resolution and clipped at twice the rms noise. Then regions of $\mathrm{H}$ I emission were retained in this cube only if the feature appeared in at least three adjacent velocity channels. The resulting cube was applied as a blanking mask to the higher resolution cubes to produce two final full-resolution subcubes: one made with natural weighting and spatial resolution $=16.7^{\prime \prime} \times 16.0^{\prime \prime}(\mathrm{FWHM})$ and referred to hereafter as the low resolution cube; the other with uniform weighting and spatial resolution $=12.4^{\prime \prime} \times 11.7^{\prime \prime}$ (FWHM) and referred to hereafter as the high resolution cube. Moment maps made from these cubes will be denoted low resolution and high resolution, respectively. Additionally, we blanked regions in the first and second moment images where the $\mathrm{H}$ I surface brightness was low in the zeroth moment image at the same resolution. Both final subcubes have pixel size $=3^{\prime \prime}$, channel width $=5.3 \mathrm{~km} \mathrm{~s}^{-1}$, and dimensions $256 \times 256 \times 58(\mathrm{RA} \times \mathrm{DEC} \times$ velocity).

The most extended $\mathrm{HI}$ emission is faint emission $4.6^{\prime}$ from the phase center, whereas the primary beam is $32^{\prime}$ (FWHM); thus, over most of the field of interest, primarybeam attenuation is negligible. We measure a total line flux for the galaxy pair of $33.3 \mathrm{Jy} \mathrm{km} \mathrm{s}{ }^{-1}$, corresponding to an $\mathrm{H}$ I mass of $2.0 \times 10^{10}\left(80 / H_{0}\right)^{2} M_{\odot}$, which is the same as obtained in the single-dish observations by Bothun \& Schommer (1982). Separation of the H I contributions of the two galaxies is not obvious because the tidal bridge of NGC 2535 overlaps the small galaxy NGC 2536 along line-ofsight and in velocity; this will be discussed in Sec. 5.7.

After eliminating the data affected by solar interference, two cleaned images of the $\lambda 20 \mathrm{~cm}$ radio continuum emission were made from the averaged, line-free uv data: one with natural weighting and spatial resolution $=15.1^{\prime \prime} \times 14.6^{\prime \prime}$ (FWHM) and the other with uniform weighting and spatial resolution $=12.0^{\prime \prime} \times 11.5^{\prime \prime}(\mathrm{FWHM})$.

Table 2 gives a summary of the $\mathrm{HI}$ observations and Table 3, the $\mathrm{H} \mathrm{I}$ and radio continuum map parameters. 
TABLE 2. VLA H I observations.

\begin{tabular}{lcc}
\hline \multicolumn{1}{c}{ Parameter } & C Configuration & D Configuration \\
\hline Observing Date & 1993 July 11 & 1993 October 30 \\
On-source Observing Time & 4 hours & 1 hour \\
Largest Scale Visible to VLA & $\mathbf{7}^{\prime}$ & $15^{\prime}$ \\
Primary Beam (FWHM) & $32^{\prime}$ & $3^{\prime}$ \\
Total Bandwidth (MHz) & & \\
IFs AC & 1.5625 & 1.5625 \\
IFs BD & 1.5625 & 1.5625 \\
Central Velocity $\left(\mathrm{km} \mathrm{s}^{-1}\right)$ & & \\
IFs AC & 4221.185 & 4221.185 \\
IFs BD & 3967.047 & 3967.047 \\
Channel width $\left(\mathrm{km} \mathrm{s}^{-1}\right)$ & 5.30 & 5.30 \\
Number of Channels & & \\
IFs AC & 64 & 64 \\
IFs BD & 64 & 64 \\
\hline \hline
\end{tabular}

3. OPTICAL AND NEAR-INFRARED MORPHOLOGY

Figure 1 shows that NGC 2535 has an inner eye-shaped oval with bright blue knots at the two apices and along the southern rim, and two long arms that depart from the eastern and western ends of the oval, respectively. On the concave side of the northern arm, near the oval, there is a short spur pointing towards the NW, and diametrically opposite on the concave side of the southern arm, a short spur pointing towards the SE (although dust lanes confuse the situation here). The contour in Fig. 1 corresponds to our limiting surface brightness, approximately 26.5-27.0 mag $\operatorname{arcsec}^{-2}$, and traces emission from the extended stellar envelope of NGC 2535 , i.e., beyond the Holmberg radius. This is unlike the situation in the ocular galaxy IC 2163 , where almost all of the stars in the outer disk have collected in the tidal arms. In Fig. 1 the outer isophote of NGC 2535 appears elliptical, with a semi-major axis of $\sim 2^{\prime}$ (compared to the isophotal radius $R_{25}$ of $1.25^{\prime}$ ), a major axis position angle of $\sim 165^{\circ}$, and an axis ratio of $2: 1$. (The semimajor axis was measured on the anticompanion side.) The isophotal major axis of the eye-shaped oval has a very different PA of $100^{\circ} \pm 4^{\circ}$ (see values listed in Table 4). The shapes and relative orientations of the eye-shaped oval and the outer isophotal ellipse suggest (a) that one or both of these features are intrinsically elliptical (as a result of the tidal interaction) or (b) that these features are significantly inclined with respect to each other and to the plane of the sky.

In a higher resolution photograph in Arp (1969), one can see prominent dust lanes along the inside edges of the main northern and southern arms, and a dust lane along the outside
TABLE 4. Orientations.

\begin{tabular}{lr}
\hline \multicolumn{1}{c}{ Parameter } & Value \\
\hline NGC 2535 & \\
Minor Axis Position Angle of Disk & \\
$\quad$ Kinematic (Optical and HI) & $63^{\circ} \pm 5^{\circ}$ \\
$\quad$ Photometric on Eye-shaped Oval & $10^{\circ} \pm 4^{\circ}$ \\
Position Angle of Maximum ( $v_{\text {obs }}-v_{\text {sys }}$ ) in HI & \\
$\quad$ on Eye-shaped Oval & $131^{\circ} \pm 5^{\circ}$ \\
Isophotal Major Axis Position Angle & \\
$\quad$ Eye-shaped Oval & $100^{\circ} \pm 4^{\circ}$ \\
Bar-like Radio Continuum Feature & $100^{\circ} \pm 4^{\circ}$ \\
Near-Infrared Bar & $52^{\circ} \pm 6^{\circ}$ \\
Outer Stellar Envelope & $165^{\circ} \pm 5^{\circ}$ \\
Preferred Position Angle of Projection Line of Nodes & $153^{\circ} \pm 5^{\circ}$ \\
Adopted Disk Inclination & $23^{\circ} \pm 4^{\circ}$ \\
NGC 2536 & \\
Photometric Major Axis of Disk & $38^{\circ} \pm 5^{\circ}$ \\
Major Axis of Near-infrared Bar & $20^{\circ} \pm 3^{\circ}$ \\
\hline \hline
\end{tabular}

edge of the southern rim of the eye-shaped oval. From spectroscopy, Arp concludes that the bright object on the southwestern rim of the eye-shaped oval is a foreground star. The continuum-subtracted $\mathrm{H} \alpha$ image in Amram et al. (1989) shows significant emission from the eye-shaped oval and the two main tidal arms of NGC 2535, with the brightest emission knots at the apices. There are bright $\mathrm{H}$ II regions along the southern rim of the eye-shaped oval and on the section of the northern arm from the east clockwise around to the north. Paralleling the northern arm from the NE clockwise around to the NNW, there is a string of faint $\mathrm{H} \alpha$ emission displaced $15^{\prime \prime}-18^{\prime \prime}$ outwards (in the plane of the sky) from the main arm. This string corresponds to an $\mathrm{H} \mathrm{I}$ feature that will be described in Sec. 5.1 below.

The northern arm of NGC 2535 is the longer arm and presently on the anticompanion side. The $N$-body simulation by Klaric (1993) successfully matches the shapes of the two main optical arms of NGC 2535 with an orbit that gets NGC 2536 to its present location and reproduces the sharp bend of the northern arm near $\mathrm{RA}(1950)=08^{\mathrm{h}} 08^{\mathrm{m}} 09^{\mathrm{s}}$. The sharp bend results from the merging, at an earlier step in the simulation, of two arms with different velocities. In this model, the northern arm of NGC 2535 is the tidal tail and the southern arm, the tidal bridge. We adopt these designations.

In the near-infrared $I, J$, and $K$-band images, NGC 2535 has a weak bar with major axis at $\mathrm{PA}=52^{\circ} \pm 6^{\circ}$ that ends at the rim of the eye-shaped oval. The lower left panel of Fig. 3 displays a contour plot of NGC 2535 from the $J$-band images that we took on a night with $0.8^{\prime \prime}$ seeing. It shows the bar and

TABLE 3. H I and radio continuum maps from combined $\mathrm{C}$ and $\mathrm{D}$ configuration data

\begin{tabular}{|c|c|c|}
\hline Parameter & $\begin{array}{l}\text { Natural Weight } \\
\text { "Low Resolution" }\end{array}$ & $\begin{array}{l}\text { Uniform Weight } \\
\text { "High Resolution" }\end{array}$ \\
\hline \multicolumn{3}{|l|}{ HI Images } \\
\hline Synthesized Beam (FWHM, PA) & $16.7^{\prime \prime} \times 16.0^{\prime \prime}, 150^{\circ}$ & $12.4^{\prime \prime} \times 11.7^{\prime \prime},-4^{\circ}$ \\
\hline Conversion Factor $\left(T_{\mathrm{b}} / S\right)\left(\mathrm{K} / \mathrm{mJy}\right.$ beam $\left.^{-1}\right)$ & 2.34 & 4.29 \\
\hline Rms Noise per Channel (mJy beam ${ }^{-1}$ ) & 0.65 & 0.88 \\
\hline $\begin{array}{l}\text { Column Density in atoms } \mathrm{cm}^{-2} \\
\text { equivalent to } 100 \mathrm{Jy}^{-1} \mathrm{bem}^{-1} \mathrm{~m} \mathrm{~s}^{-1}\end{array}$ & $4.2 \times 10^{20}$ & $7.6 \times 10^{20}$ \\
\hline \multicolumn{3}{|l|}{ Radio Continuum Images } \\
\hline Synthesized Beam (FWHM, PA) & $15.1^{\prime \prime} \times 14.6^{\prime \prime},-15^{\circ}$ & $12.0^{\prime \prime} \times 11.5^{\prime \prime},-10^{\circ}$ \\
\hline Conversion Factor $\left(T_{\mathrm{b}} / S\right)\left(\mathrm{K} / \mathrm{mJy}\right.$ beam $\left.{ }^{-1}\right)$ & 2.82 & 4.51 \\
\hline Rms Noise $\left(\mathrm{mJy}_{\text {beam }}{ }^{-1}\right)$ & 0.15 & 0.19 \\
\hline
\end{tabular}



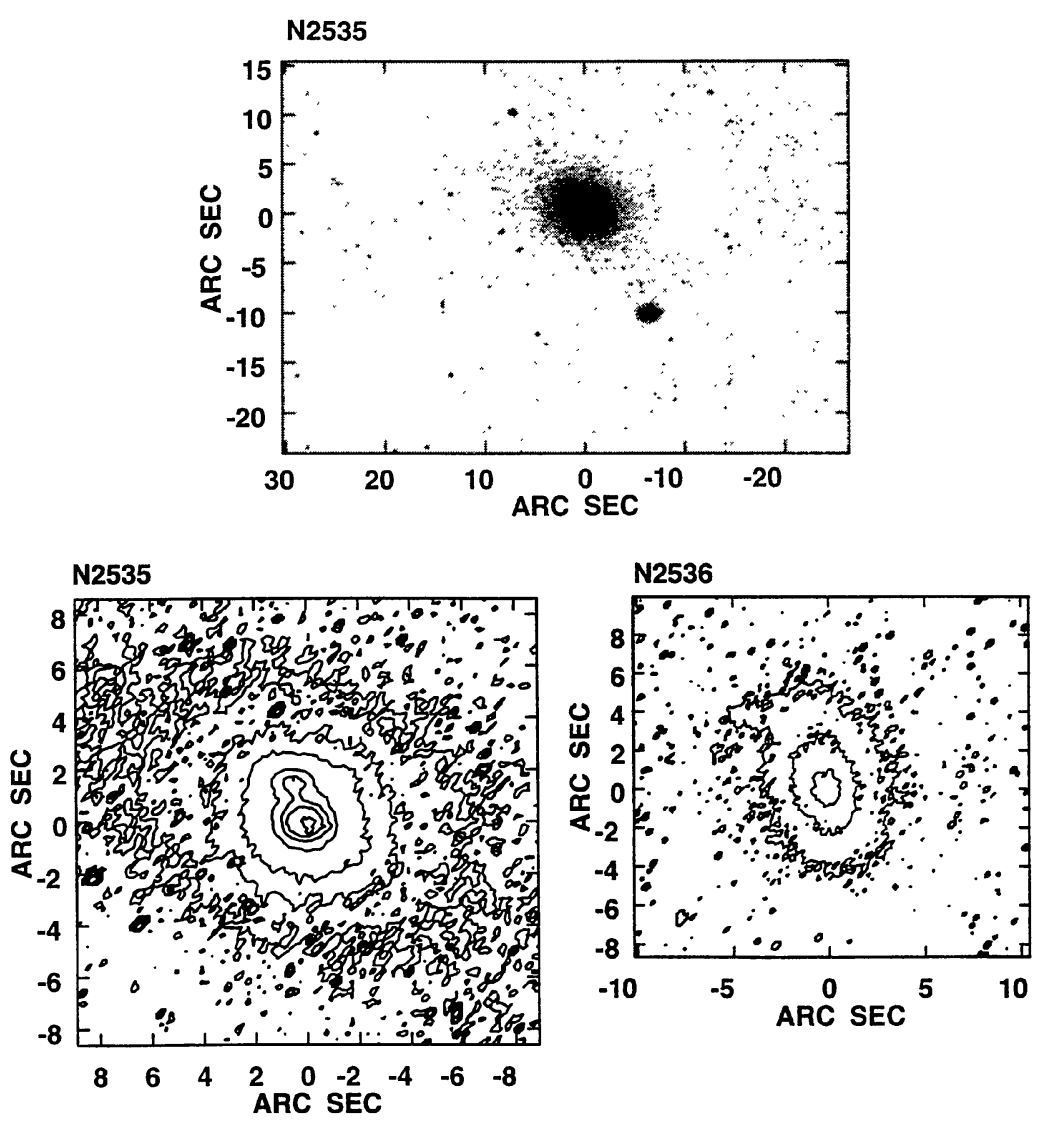

FIG. 3. Near-infrared $J$-band images of the centers of NGC 2535 (bottom left and top) and NGC 2536 (bottom right). These show a bar-like feature in the center of each galaxy.

another source about $2 " \mathrm{NNE}$ of the nucleus. This second source could be either a foreground star or a supernova in NGC 2535, but we have no data to discriminate between these possibilities. The greyscale display of the $J$-band image of NGC 2535 in the top panel of Fig. 3 includes the bar, the start of the tidal tail, the rather weak emission from the southern rim of the eye-shaped oval, and the bright foreground star 12" SW of the nucleus. The southern rim of the eye-shaped oval is more clearly outlined by the bright knots of star formation in the $B$-band image than in the nearinfrared images. For later comparison with the radio continuum emission, it is important to note that there is no stellar bar connecting the two apices of the eye-shaped oval at a PA of $100^{\circ}$.

The companion NGC 2536, located at the end of the tidal bridge of NGC 2535, has broad arms that wind in the same sense as the tidal arms of NGC 2535. If the arms in both galaxies are trailing, then both galaxies are rotating counterclockwise. From the sky-subtracted $I$-band image, we find that the luminosity of NGC 2536 is $0.26 \pm 0.01$ times the luminosity of NGC 2535. The main uncertainties in converting this to a mass ratio are: (a) NGC 2536 is undergoing a starburst so may be anomalously bright, even in $I$ band; (b) the $I$-band luminosities have not been corrected for extinction. Both galaxies have the same, rather blue, $(B-V)_{T}$ color $\approx 0.55$ (see Table 1 ). The observed value of the lumi- nosity ratio in $I$ band is similar to the value of the mass ratio (0.3) in Klarić's (1993) simulation of the galaxy encounter.

Although NGC 2536 is located at the end of the tidal bridge of NGC 2635, the blue color of NGC 2536, also evident in the picture in the Wray Atlas (Wray 1988), suggests a young population without much extinction. This is unlike the M51 system, in which the arm of M51 is clearly in front of its companion (Sandage 1961). In Klarić's model, NGC 2536 is between us and much of the bridge gas.

Amram et al. (1989) and Arp (1969) find that the brightest $\mathrm{H} \alpha$ emission from this system comes from a knot $\sim 5^{\prime \prime}$ $\mathrm{NE}$ of the center of NGC 2536. The $J$ - and $K$-band images of NGC 2536 show an inner bar with a position angle of $20^{\circ}$ $\pm 3^{\circ}$ and major axis $\times$ minor axis $=8.8^{\prime \prime} \times 5.2^{\prime \prime}$. The lower right panel in Fig. 3 displays a contour plot of the $J$-band emission from the center of NGC 2536. In addition to the bar, there is an extension from the northern part of the bar towards the east $\left(\sim 5^{\prime \prime}-6^{\prime \prime} \mathrm{NE}\right.$ of the nucleus). This extension coincides with the optical emission-line knot.

\section{CO RESULTS}

Figure 4 displays the set of line profiles at the 12 positions where we searched for ${ }^{12} \mathrm{CO} J=1 \rightarrow 0$ emission. The positions are distributed on a grid with one beam (33") spacing and $\mathrm{PA}=49^{\circ}$ (except for positions $\mathrm{CH}$ and $\mathrm{EI}$, which are 

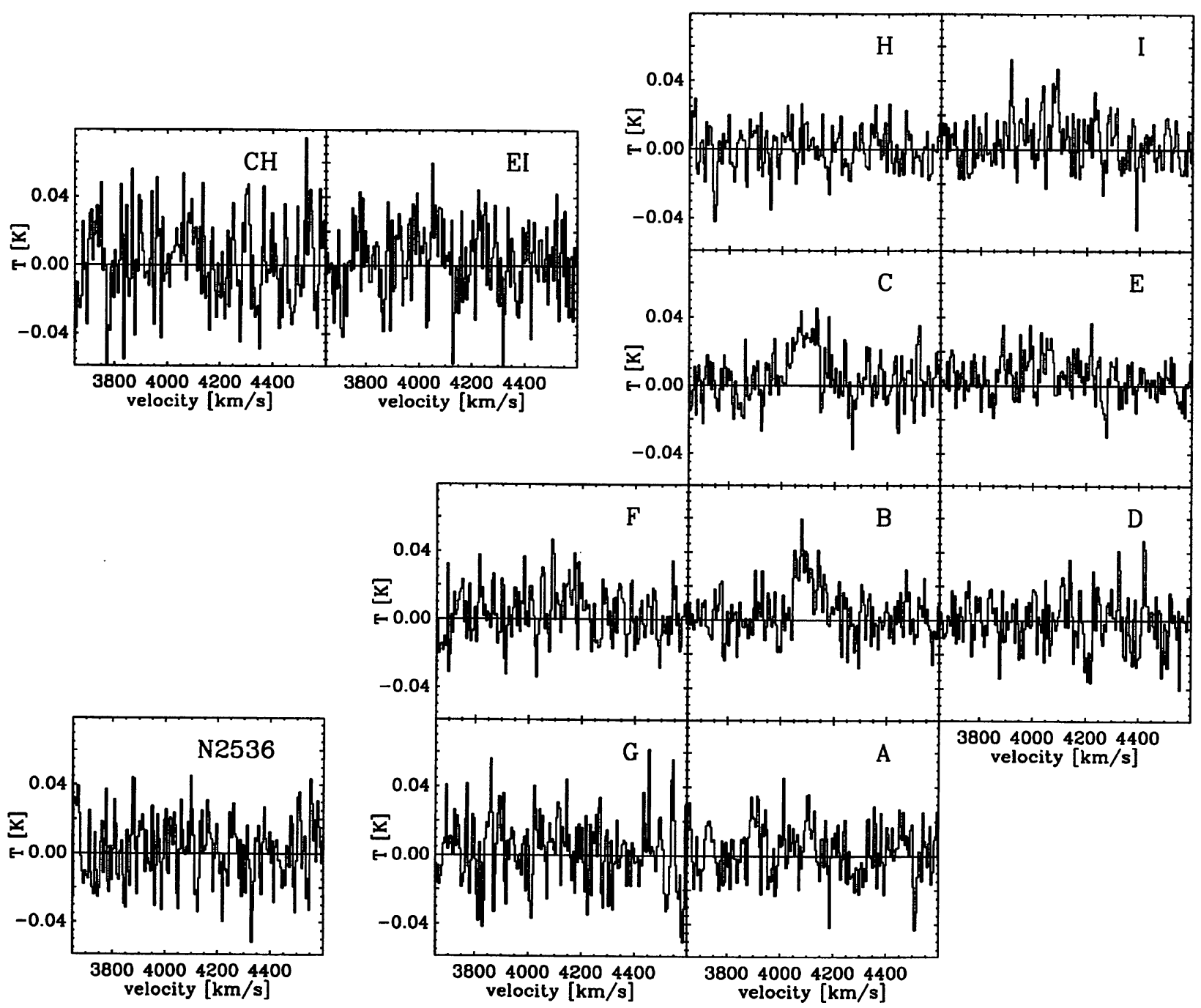

FIG. 4. The ${ }^{12} \mathrm{CO}$ line profiles. The label in each panel corresponds to the position where the spectrum was taken; these are marked in Fig. 5. The abscissa is the heliocentric velocity, optical definition. The intensities are given as main beam brightness temperature, $T_{\mathrm{mb}}$ (kelvin).

found between positions $\mathrm{C}$ and $\mathrm{H}$, and $\mathrm{E}$ and $\mathrm{I}$, respectively); they are marked on the greyscale display of the $B$-band image in the top panel of Fig. 5. The circles indicate the locations of two clear detections, one (position B) centered on NGC 2535 and the other (position C) at $\operatorname{RA}(1950)=08^{\mathrm{h}} 08^{\mathrm{m}} 14.64^{\mathrm{s}}, \operatorname{DEC}(1950)=25^{\circ} 21^{\prime} 41^{\prime \prime}$, close to the center of NGC 2535 but on its tidal tail.

The central position (position B) has an integrated intensity $I_{\mathrm{CO}}=\int T_{\mathrm{mb}} d V$ of $3.6 \mathrm{~K} \mathrm{~km} \mathrm{~s}^{-1}$ and a peak $T_{\mathrm{mb}}$ of $0.059 \pm 0.012 \mathrm{~K}$ at a velocity of $4077 \mathrm{~km} \mathrm{~s}^{-1}$. The equivalent width $I_{\mathrm{CO}}$ (peak $T_{\mathrm{mb}}$ ) is $61 \mathrm{~km} \mathrm{~s}^{-1}$. The $\mathrm{CO}$ line profile is asymmetric. A Gaussian fit gives an amplitude of $0.036 \mathrm{~K}$, a center velocity of $4095 \mathrm{~km} \mathrm{~s}^{-1}$, and a FWHM linewidth of $97 \mathrm{~km} \mathrm{~s}^{-1}$. The spatial resolution of the ${ }^{12} \mathrm{CO}$ data is too low to tell whether the ${ }^{12} \mathrm{CO}$ emission forms an eye-shaped oval like the optical emission. The $\mathrm{H} \alpha$ velocity field here spans the range from $4025 \mathrm{~km} \mathrm{~s}^{-1}$ in the northwest to $4165 \mathrm{~km} \mathrm{~s}^{-1}$ in the southeast (Amram et al. 1989). Comparison between the $\mathrm{CO}$ profiles and the $\mathrm{H} \mathrm{I}$ line profiles from a cube con- volved to the same spatial resolution as the $\mathrm{CO}$ data will be made in Sec. 5.3.

Position $\mathrm{C}$ has $I_{\mathrm{CO}}=3.8 \mathrm{~K} \mathrm{~km} \mathrm{~s}^{-1}$ and peak $T_{\mathrm{mb}}=0.045$ $\pm 0.013 \mathrm{~K}$. The profile at position $\mathrm{C}$ is more symmetric than at position B. A Gaussian fit gives an amplitude of $0.032 \mathrm{~K}$, a center velocity of $4088 \mathrm{~km} \mathrm{~s}^{-1}$, and a FWHM linewidth of $119 \mathrm{~km} \mathrm{~s}^{-1}$ (the equivalent width calculated as above is $85 \mathrm{~km} \mathrm{~s}^{-1}$ ). The $\mathrm{H} \alpha$ velocity field here goes from 4035 to $4135 \mathrm{~km} \mathrm{~s}^{-1}$. If we adopt the conversion factor $N\left(\mathrm{H}_{2}\right)=2.3$ $\times 10^{20} I_{\mathrm{CO}} \mathrm{H}_{2} \mathrm{~cm}^{-2}$ from Strong et al. (1988), then the $\mathrm{H}_{2}$ mass is $9.6 \times 10^{8} \mathscr{M b}_{\odot}$ for position $\mathrm{B}$ and $1.0 \times 10^{9} \mathscr{C l}_{\odot}$ for position $\mathrm{C}$; the average $\mathrm{H}_{2}$ column densities are $13-14 \mathscr{M b}_{\odot} \mathrm{pc}^{-2}$.

Aside from the clear detections at positions B and C, we have a weak detection at position I with amplitude $3 \times$ the rms noise. At positions $\mathrm{A}$ and $\mathrm{E}$, we have possible detections with amplitudes $2 \times$ the rms noise. At all the other positions the amplitudes are below $2 \times$ the rms noise. Gaussian fits give the following values for the amplitude, center velocity, 

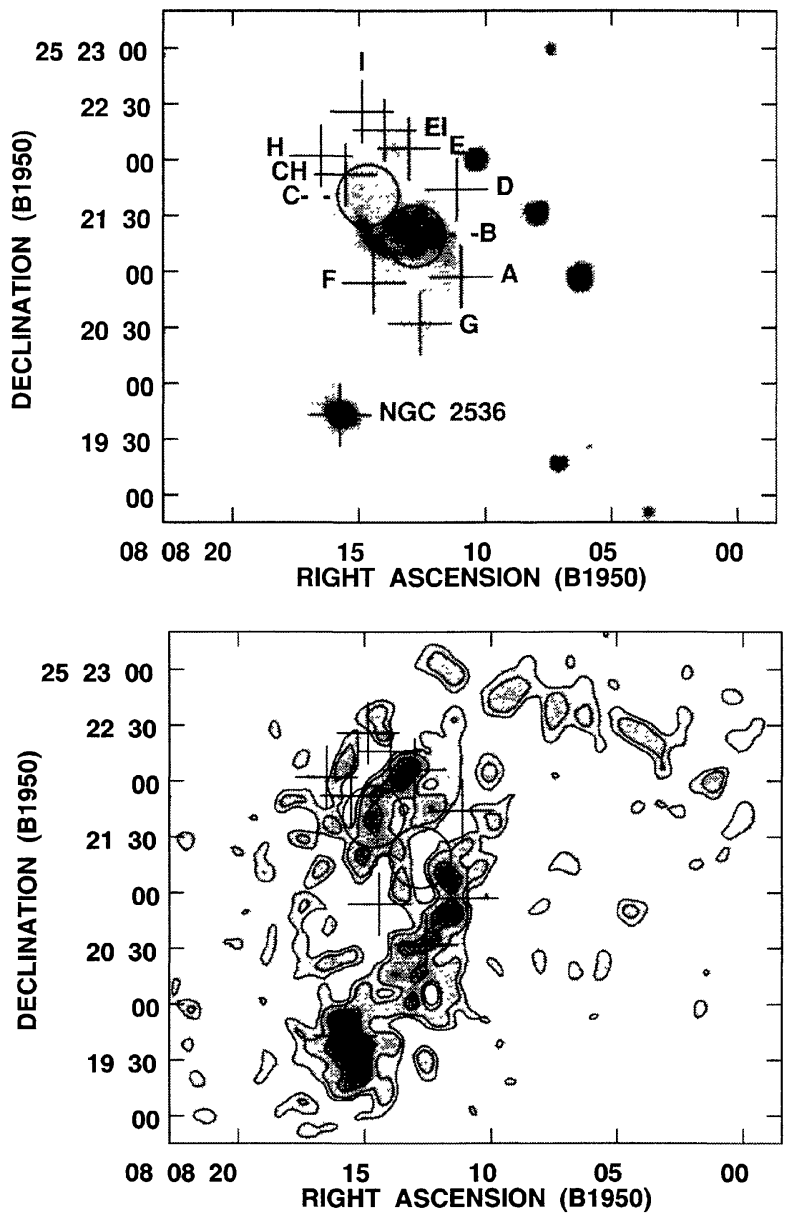

Fig. 5. Top: Greyscale display of the optical $B$-band image with symbols, equal in size to the ${ }^{12} \mathrm{CO}$ aperture, marking the 12 positions where we searched for ${ }^{12} \mathrm{CO}(1-0)$ emission. The circles mark the two clear detections and the plus signs, the 10 other positions. Bottom: Greyscale plus contour display of the high resolution $N(\mathrm{HI})$ image (resolution $\left.=12.4^{\prime \prime} \times 11.7^{\prime \prime}\right)$ for the two galaxies combined. The same ${ }^{12} \mathrm{CO}$ positions are marked as in the top panel. The contours are at $4.6,7.6,14,18$, and $21 \times 10^{20}$ atoms $\mathrm{cm}^{-2}$.

and FWHM linewidth for positions $\mathrm{A}, \mathrm{E}$, and I: position A: $0.030 \mathrm{~K}, 4108 \mathrm{~km} \mathrm{~s}^{-1}, 30 \mathrm{~km} \mathrm{~s}^{-1}$; position E: $0.023 \mathrm{~K}$, $4053 \mathrm{~km} \mathrm{~s}^{-1}, 47 \mathrm{~km} \mathrm{~s}^{-1}$; position I: $0.040 \mathrm{~K}, 4081 \mathrm{~km} \mathrm{~s}^{-1}$, $27 \mathrm{~km} \mathrm{~s}^{-1}$. This gives an $\mathrm{H}_{2}$ mass of $3 \times 10^{8} \mathscr{M} \odot$ for position I and $2-3 \times 10^{8} \mathscr{Q}_{\odot}$ for each of positions $\mathrm{A}$ and E. For positions $A$ and $E$, the $\mathrm{H}_{2}$ masses should be regarded as upper limits since the amplitudes are only $2 \times$ the rms noise.

We failed to detect ${ }^{12} \mathrm{CO}$ emission from the starburst galaxy NGC 2536 (with an rms noise of 0.019 K; see Fig. 4). If the CO linewidth (FWHM) were equal to the entire $110 \mathrm{~km} \mathrm{~s}^{-1}$ range of $\mathrm{H} \alpha$ velocities in the $\mathrm{CO}$ aperture and if the above value for the conversion factor applies to NGC 2536 (note, we have no information about the metallicity of this galaxy), then this would place an upper limit of 6 $\times 10^{8} \mathscr{L}_{\odot}$ on the $\mathrm{H}_{2}$ mass of NGC 2536 .

\section{H I RESULTS}

\subsection{General H I Morphology of the System}

For the two galaxies combined, the bottom panel of Fig. 5 displays the $N(\mathrm{H} \mathrm{I})$ image made from the high resolution cube $\left(12.4^{\prime \prime} \times 11.7^{\prime \prime}\right)$ as greyscale plus contours, and the top panel of Fig. 6 (Plate 102) displays the H I column density $N(\mathrm{H} \mathrm{I})$ image made from the low resolution cube (16.7" $\left.\times 16.0^{\prime \prime}\right)$ in greyscale. Throughout this paper, $N(\mathrm{H} I)$ denotes the line-of-sight column density, and we assume the $\mathrm{HI}$ emission is optically thin. A surface brightness of $100 \mathrm{Jy} \mathrm{beam}^{-1} \mathrm{~m} \mathrm{~s}^{-1}$ corresponds to a column density of $4.2 \times 10^{20}$ atoms cm $\mathrm{cm}^{-2}$ or $3.3 \mathscr{M} \odot \mathrm{pc}^{-2}$ in the low resolution image and to $7.6 \times 10^{20}$ atoms $\mathrm{cm}^{-2}$ or $6.1 \mathscr{L}_{\odot} \mathrm{pc}^{-2}$ in the high resolution image. The bottom panel of Fig. 6 presents the $B$-band image in greyscale with contours from the high resolution $N(\mathrm{H} \mathrm{I})$ image and with labels on features of interest. Numbered boxes are drawn around five unusually massive clouds that will be discussed in Sec. 5.5 below. In Fig. 7 contours from the low resolution $N(\mathrm{H} \mathrm{I})$ image are overlaid on the $B$-band image in greyscale with a transfer function that emphasizes the fainter blue emission.

The prominent $\mathrm{H}$ I features of NGC 2535 are the tidal tail, the tidal bridge, and an outer, elliptically-shaped arc. The eye-shaped oval seen in the $B$-band image is not present as an $\mathrm{H}$ I feature. In fact, the nucleus of NGC 2535 is located in an $\mathrm{H}$ I trough filled in with ${ }^{12} \mathrm{CO}$ emission (see Fig. 5). The portion of the $\mathrm{H}$ I elliptical arc from due east of the nucleus around clockwise to the north to $\operatorname{RA}(1950)=08^{\mathrm{h}} 08^{\mathrm{m}} 12^{\mathrm{s}}$ lies parallel, in projection, to the tidal tail and is displaced about $25^{\prime \prime}=6 \mathrm{kpc}$ (plane of sky) beyond the ridge on the main $\mathrm{H} \mathrm{I}$ arm. From the NE around clockwise to the N, the elliptical arc coincides with the string of faint $\mathrm{H} \alpha$ emission paralleling the main arm in Amram et al. (1989). The elliptical arc joins or crosses the tidal tail (in projection) just before the latter has a sharp bend. On the western side of NGC 2535, there seems to be a weaker continuation of the elliptical arc from the crossing point on the tidal tail to a position west of the western apex of the eye-shaped oval. One can imagine that the elliptical arc and this weaker extension form an ellipse that can be traced from the west counterclockwise around to the southeast, ending somewhat north of NGC 2536. It is somewhat off-centered relative to the nucleus of NGC 2535 , and its major axis has a position angle of $172^{\circ} \pm 5^{\circ}$. Its continuation on the western side will be clearer when we look at the velocity field in Sec. 5.2.2. The elliptical arc is not apparent as a feature in the $B$-band image but is similar in elliptical shape and orientation to the outer isophote of the stellar disk in the $B$-band image.

For the most part, the bright $\mathrm{H}$ I arms and the main optical arms coincide. In the north, the $\mathrm{H}$ I arm and the optical arm have a sharp bend at the same place. However, from Fig. 7, one can see that just after leaving the apex of the oval, the $\mathrm{H}$ I ridge on the tidal tail lies along the inside edge of the optical arm (or between the main arm and the spur); the $\mathrm{H} \mathrm{I}$ ridge probably coincides with the dust lane there.

The two galaxies are embedded in a large $\mathrm{H}$ I envelope about $3.3^{\prime}=48 \mathrm{kpc}$ in radius (see Fig. 6). In the low resolution image, the $\mathrm{H}$ I column density just beyond the elliptical arc is $2.5 \times 10^{20}-4.2 \times 10^{20}$ atoms $\mathrm{cm}^{-2}$. We assume that the $\mathrm{H}$ I envelope is associated with the larger galaxy. Time-scale arguments indicate that the outer $\mathrm{H}$ I envelope predates the encounter as there is not sufficient time to get the gas out to $48 \mathrm{kpc}$ in the $100 \mathrm{Myr}$ since perigalacticon in Klaric's model 


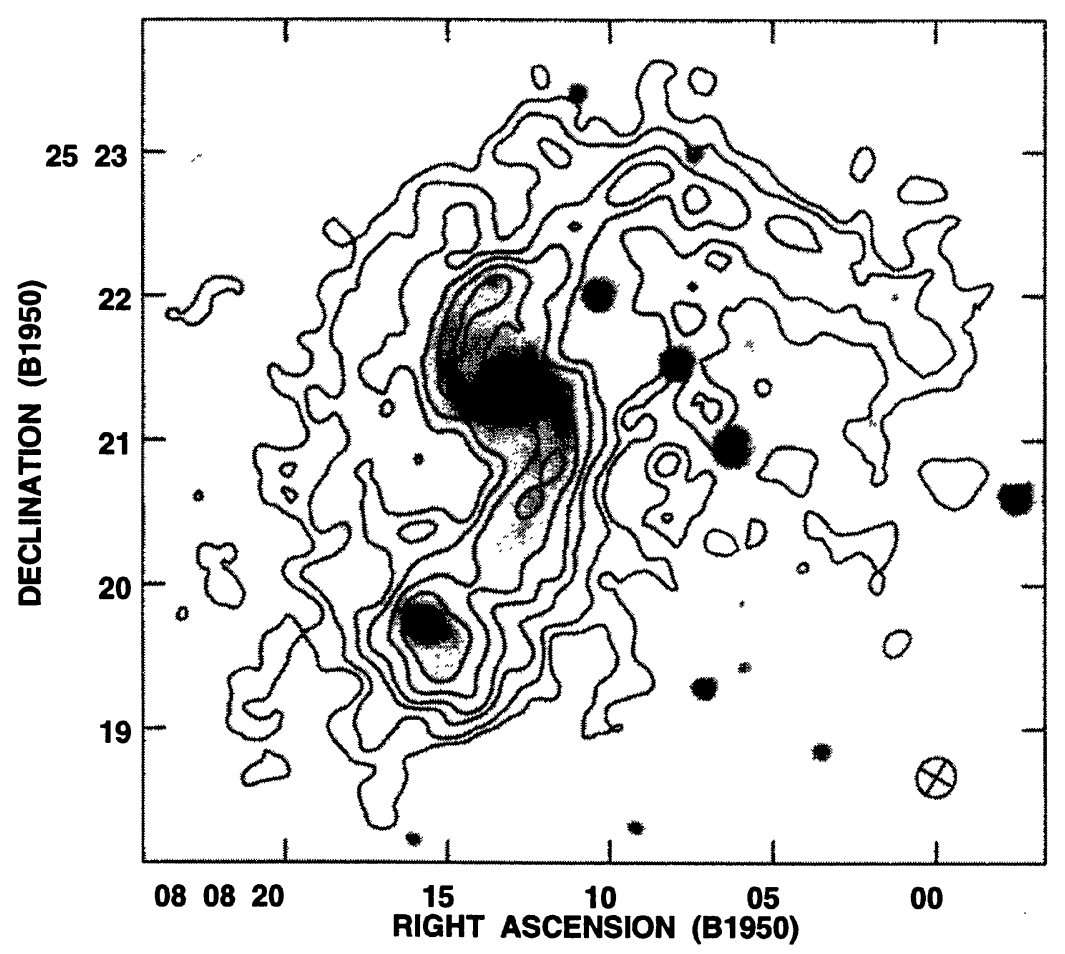

FIG. 7. Greyscale display of the $B$-band image with $N(\mathrm{H}$ I) contours from the low resolution data overlaid. The beam symbol represents the $\mathrm{H}$ I resolution $\left(16.7^{\prime \prime} \times 16.0^{\prime \prime}\right)$. The contour levels are at $2.5,4.2,7.5,10,14,18$, and $27 \times 10^{20}$ atoms cm${ }^{-2}$. Notice the approximate correspondence between the extent of the blue-light emission and the $N(\mathrm{HI})$ contour at $4.2 \times 10^{20}$ atoms $\mathrm{cm}^{-2}$.

(1993). Figure 7 shows that the outer isophote of the stellar disk in our $B$-band image coincides roughly with the $4.2 \times 10^{20}$ atoms $\mathrm{cm}^{-2}$ contour in the low resolution $N(\mathrm{H} \mathrm{I})$ image. The $\mathrm{H}$ I envelope is more extensive than the stellar envelope in our images.

NGC 2536 is located in an H I clump with mass $2 \times 10^{9} \mathscr{C}_{\odot}$ at the southern end of the tidal bridge of NGC 2535 . The clump consists of bridge gas plus gas associated with NGC 2536 and will be discussed in Sec. 5.7 below. At our highest H I spatial resolution, the optical major axis of NGC 2536 is only about 3 synthesized beamwidths.

\subsection{Velocity Field and Velocity Streaming}

The bottom panel of Fig. 8 is a greyscale plus contour display of the velocity field made from the low resolution $\left(16.7^{\prime \prime} \times 16.0^{\prime \prime}\right)$ cube. These isovelocity contours are overlaid on the high resolution $N(\mathrm{HI})$ image in greyscale in the top panel of Fig. 8 and on the $B$-band image in greyscale in the top panel of Fig. 9. In these images, velocity information is restricted to areas of sufficiently high $\mathrm{H}$ I surface brightness. The bottom panel of Fig. 9 displays a more sensitive $N(\mathrm{H} \mathrm{I})$ image and the global $\mathrm{H}$ I velocity field, both derived from a cube convolved to $30^{\prime \prime}$ resolution. In this panel, velocity contours are drawn only where $N(\mathrm{H} \mathrm{I})$ exceeds $1.0 \mathscr{L}_{\odot} \mathrm{pc}^{-2}$. Figure 10 presents a set of channel maps (every third channel with emission in excess of $3 \times$ the rms noise) from the cube with $16.7^{\prime \prime} \times 16.0^{\prime \prime}$ resolution overlaid on the $B$-band image in greyscale.

From Fabry-Perot $\mathrm{H} \alpha$ observations, Amram et al. (1989) obtain a velocity field at higher spatial resolution: for NGC
2535 , they find $v$ (sys) $=4095 \pm 10 \mathrm{~km} \mathrm{~s}^{-1}$ with kinematic minor axis at $63^{\circ} \pm 5^{\circ}$; for NGC 2536, they find $v$ (sys) $=4085 \pm 10 \mathrm{~km} \mathrm{~s}^{-1}$ with isophotal major axis at $38^{\circ}$. The H I velocity field of NGC 2535 gives $v$ (sys) $=4100 \pm 5 \mathrm{~km} \mathrm{~s}^{-1}$ and kinematic minor axis $\mathrm{PA}=62^{\circ} \pm 2^{\circ}$, consistent with the optical values. The receding side of the eye-shaped oval in NGC 2535 is in the SE. For trailing arms, NGC 2535 is rotating counterclockwise and the near side is on the NE kinematic minor axis.

The situation for NGC 2536 is more complicated and will be discussed in Sec. 5.7.

\subsubsection{Oval shape of central disk of NGC 2535}

The eye-shaped oval of NGC 2535 has a $53^{\circ} \pm 5^{\circ}$ misalignment between its kinematic minor axis at $63^{\circ}$ and its photometric minor axis at $10^{\circ}$ (see Table 4). Paper I finds a similar misalignment in IC 2163 and uses it to argue for an intrinsically oval shape to the central disk. The same interpretation applies to NGC 2535; this is supported by the following comparison between the luminosity of the galaxy and maximum value of $\left|v_{\text {obs }}-v_{\text {sys }}\right|$ before deprojection (we call this the "projected maximum velocity").

The projected maximum velocity is $75 \mathrm{~km} \mathrm{~s}^{-1}$ in the $\mathrm{H} \alpha$ velocity field and $45 \mathrm{~km} \mathrm{~s}^{-1}$ in the $\mathrm{H}$ I velocity field; it occurs just beyond the eye-shaped oval. We adopt the optical value (spatial resolution $=1.5^{\prime \prime}$ ) because it is likely that the $\mathrm{H}$ I value has been reduced by beam smearing. The $B$ magnitude of NGC 2535, corrected for extinction in our galaxy is $13.17 \pm 0.13$, equivalent to $M_{B}=-20.33 \pm 0.13$ and $L_{B}$ $=2.0 \times 10^{10} L_{B \odot}$ if $H_{0}=80 \mathrm{~km} \mathrm{~s}^{-1} \mathrm{Mpc}^{-1}$. To err on the 

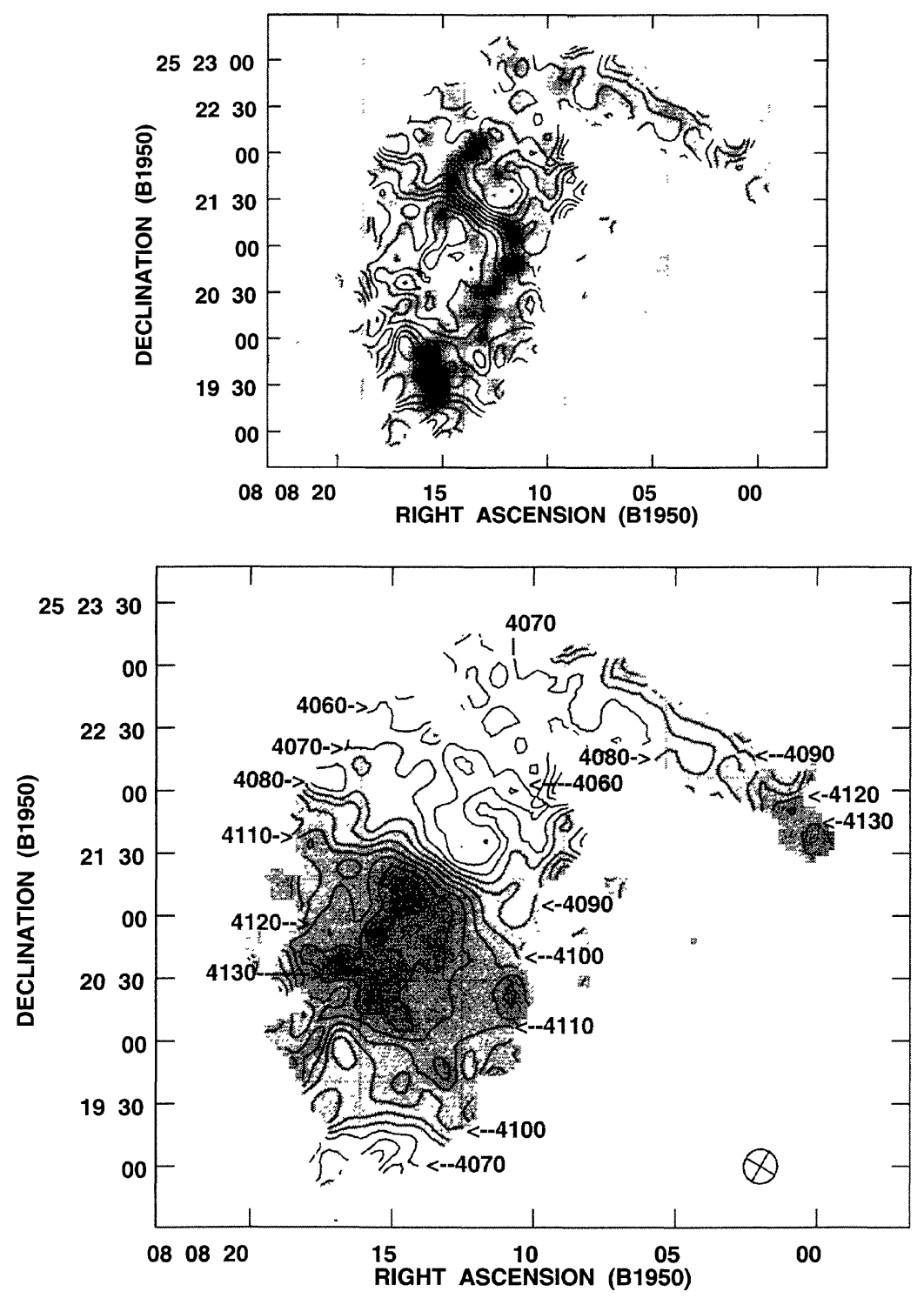

FIG. 8. Bottom: Greyscale and contour display of the $\mathrm{H}$ I velocity field made from the low resolution $\left(16.7^{\prime \prime} \times 16.0^{\prime \prime}\right)$ cube. Top: The same $\mathrm{H}$ I isovelocity contours overlaid on the high resolution $N(\mathrm{H} \mathrm{I})$ image in greyscale. The contour interval is $10 \mathrm{~km} \mathrm{~s}^{-1}$.

conservative side, we have omitted the correction for internal extinction. From a comparison of rotation curves and luminosities of undisturbed spiral galaxies, Persic \& Salucci (1991) find $v_{\max }=200\left(L_{B} / L_{B}^{*}\right)^{1 / 4} \mathrm{~km} \mathrm{~s}^{-1}$, where (after adjustment to $\left.H_{0}=80 \mathrm{~km} \mathrm{~s}^{-1} \mathrm{Mpc}^{-1}\right) L_{B}^{*}=2.3 \times 10^{10} L_{B \odot}$ and corresponds to a $B$ magnitude of -20.5 . There are several uncertainties in applying this relation to NGC 2535: the observed velocities may include streaming motions, and NGC 2535 has somewhat enhanced star formation, but not a starburst (Bernlöhr 1993). For an undisturbed spiral with the same luminosity as NGC 2535, the Persic-Salucci relation predicts $v_{\max }=193 \mathrm{~km} \mathrm{~s}^{-1}$. If $v_{\max }=193 \mathrm{~km} \mathrm{~s}^{-1}$ and the observed value is $75 \mathrm{~km} \mathrm{~s}^{-1}$, then the inclination $i=23^{\circ} \pm 4^{\circ}$ (where $i=0^{\circ}$ for a face-on galaxy). The estimated uncertainty in $i$ allows for streaming motions before deprojection of $\pm 10 \mathrm{~km} \mathrm{~s}^{-1}$ (see Sec. 5.2.2) and an uncertainty in $M_{B}$ of $\pm 0.5 \mathrm{mag}$. Assuming that the eye-shaped oval is intrinsically circular, Amram et al. (1989) deduce $i=43^{\circ} \pm 10^{\circ}$ from the isophotes and thus conclude that the maximum circular velocity is $110 \mathrm{~km} \mathrm{~s}^{-1}$, well below the value implied by the Persic-Salucci expression for undisturbed spirals. So either NGC 2535 is anomalously bright by a factor of 10 (which would put it in the starburst category) or the central disk has an intrinsically oval shape and $i$ is appreciably less than $43^{\circ}$. Another argument in favor of the latter option is that a $v_{\max }$ of $110 \mathrm{~km} \mathrm{~s}^{-1}$ would result in a total (dynamical) mass for NGC 2535 of $2.5-5 \times 10^{10} \mathscr{M}_{\odot}$, which is low, especially when compared to the observed $\mathrm{HI}$ mass of $9 \times 10^{9} \mathscr{C b}_{\odot}$ within the same region (i.e., out to the $105^{\prime \prime}$ apparent distance of the companion).

In summary, the large misalignment between the kine- 

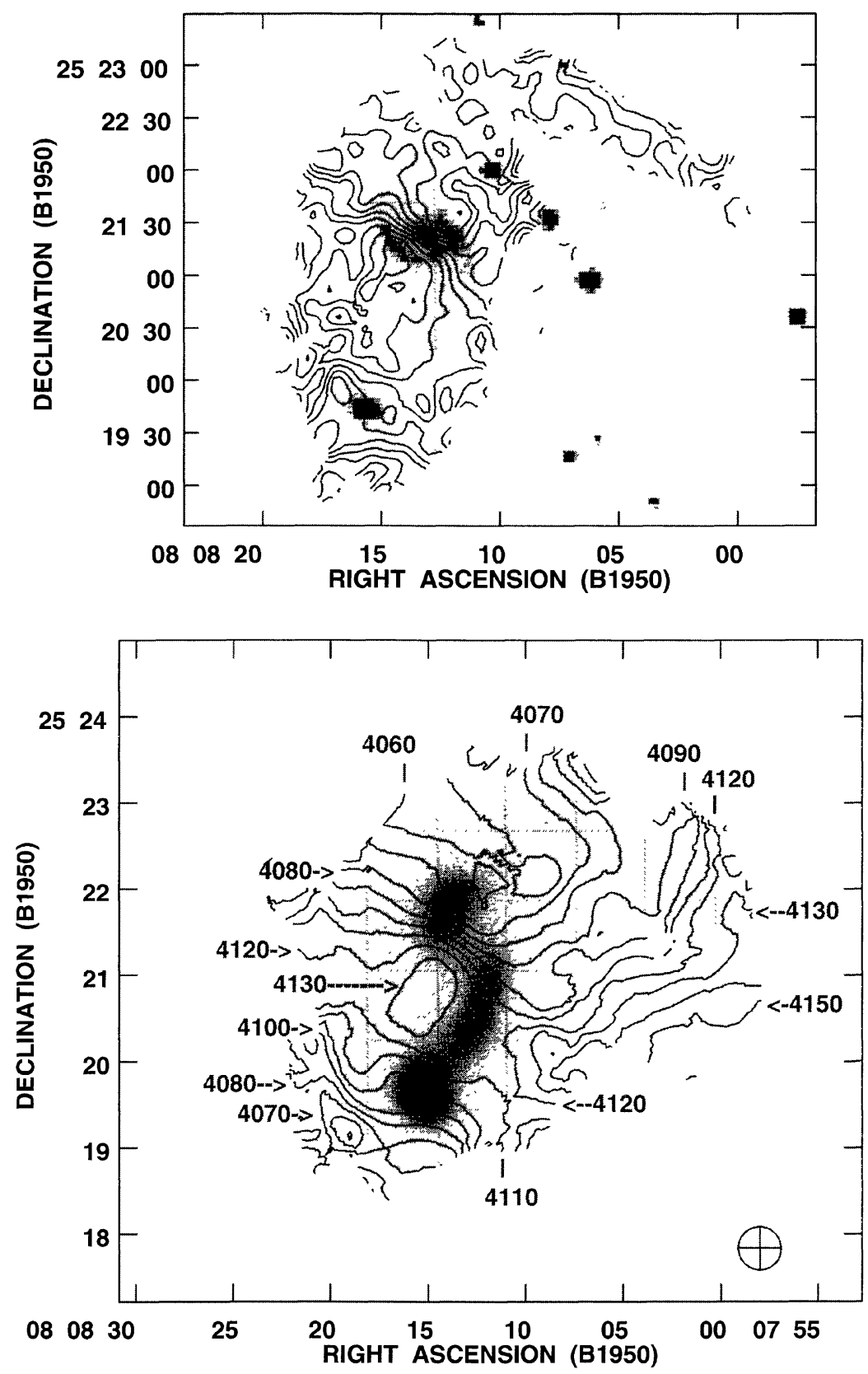

FIG. 9. Top: $\mathrm{H}$ I isovelocity contours from Fig. 8 overlaid on the $B$-band image in greyscale. Bottom: Isovelocity contours of the global $\mathrm{H}$ I velocity field overlaid on the $N(\mathrm{HI})$ image in greyscale, both made from a cube with $30^{\prime \prime}$ resolution. The contour interval is $10 \mathrm{~km} \mathrm{~s}^{-1}$.

matic minor axis and the photometric minor axis of the eyeshaped oval and the comparison between the luminosity of the galaxy and the projected maximum velocity imply that the eye-shaped oval is intrinsically oval and that we are seeing the central disk close to face-on. In the rest of this paper, we adopt the following values for the projection parameters of the central disk of NGC 2535: inclination $i=23^{\circ} \pm 4^{\circ}$ and position angle of the line of nodes of the projection (the intersection between the plane of the disk and the plane of the sky) $=153^{\circ}$, i.e., perpendicular to the kinematic minor axis. Note that this position angle is approximately the same as the position angle of the photometric major axis of the outer isophotal ellipse marked in the $B$-band image in Fig. 1 . Since this outer isophotal ellipse has an axis ratio of $2: 1$, the outer stellar disk is either intrinsically elliptical or it is tilted along the kinematic minor axis by $\simeq 40^{\circ}$ relative to the central disk.

\subsubsection{Velocity streaming in NGC 2535}

In the northeastern part of NGC 2535, the velocity field in Fig. 8 shows kinks (indicative of peculiar velocities or 


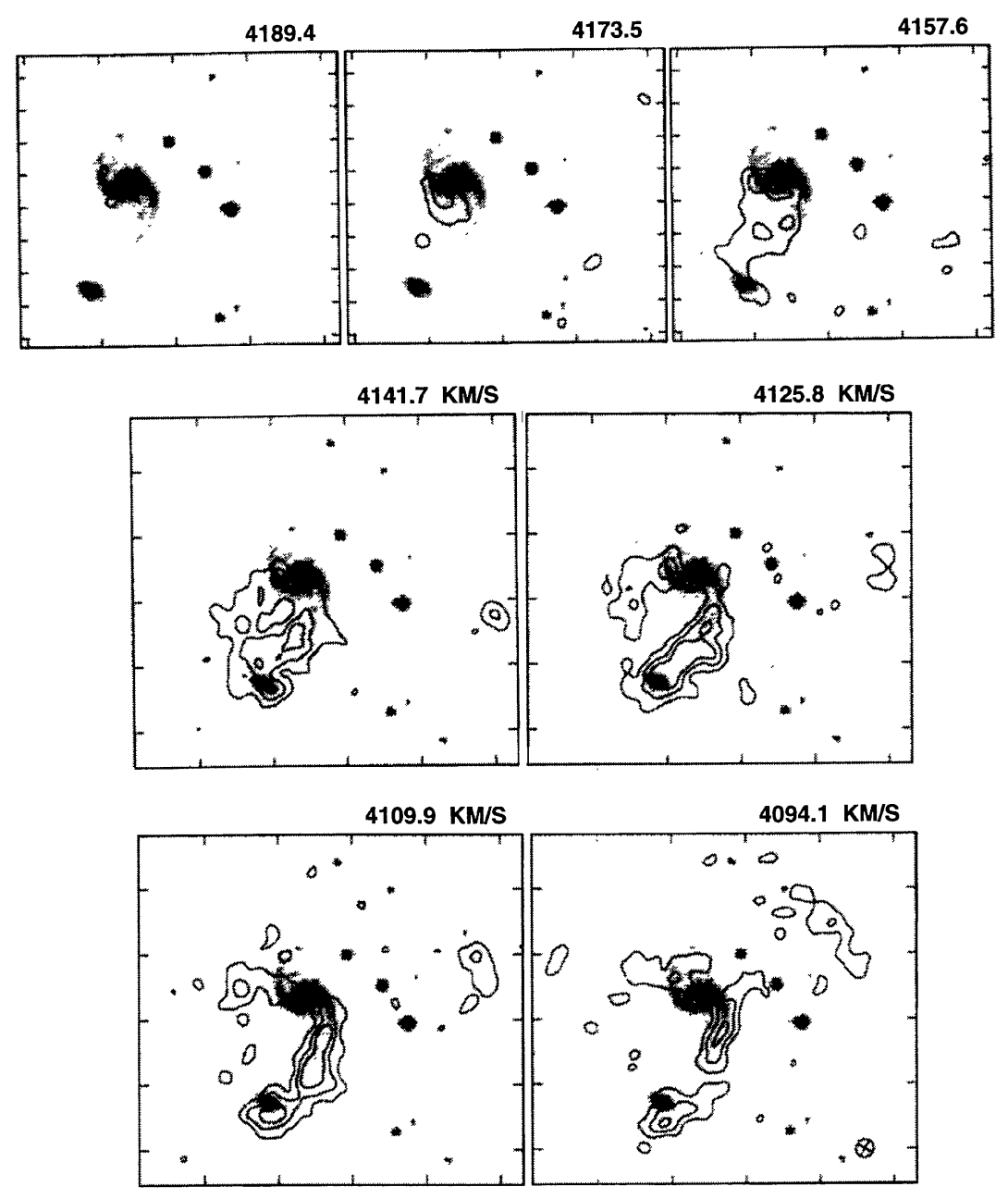

FIG. 10. A set of channel maps (every third channel map) from the $\mathrm{H}$ I cube with low resolution $\left(16.7^{\prime \prime} \times 16.0^{\prime \prime}\right)$ overlaid on a greyscale display of the $B$-band image. The symbol in the $4094 \mathrm{~km} \mathrm{~s}^{-1}$ panel represents the $\mathrm{H}$ I beam size. The rms noise is $0.65 \mathrm{mJy}$ beam ${ }^{-1}$, which corresponds to $1.5 \mathrm{~K}$, and the contour levels are at 3,6 , and $9 \times$ the rms noise.

streaming motions) where the isovelocity contours cross the tidal tail (the northern arm) and where the contours cross the elliptical arc, particularly where these two structures are parallel in projection (i.e., from due east of the nucleus clockwise around to due north). The velocity on the elliptical arc is about the same as the velocity on the parallel portion of the tidal tail at the same azimuth. On the western side of the galaxy, there are velocity kinks in the opposite sense on the apparent continuation of the elliptical arc after crossing the tidal tail in the north at $\operatorname{RA}(1950)=08^{\mathrm{h}} 08^{\mathrm{m}} 12^{\mathrm{s}}$. The velocity kinks make it easier to see the elliptical arc and its weaker continuation on the western side as a coherent ellipse with peculiar velocities approaching us on the western side of NGC 2535 and peculiar velocities moving away from us on the eastern side of NGC 2535. These projected peculiar velocities are about $10-20 \mathrm{~km} \mathrm{~s}^{-1}$.

From Fig. 8 and the channel maps in Fig. 10, one sees that the $\mathrm{H} \mathrm{I}$ velocity remains fairly constant at $4060-4070 \mathrm{~km} \mathrm{~s}^{-1}$ over a long portion of the tidal tail from massive Cloud 1 (marked in Fig. 6) until $\mathrm{PA}=-50^{\circ}$ (past the sharp bend in the arm), and then increases steadily to
$4130 \mathrm{~km} \mathrm{~s}^{-1}$. Although the $\mathrm{H} \alpha$ emission from this arm provides poorer coverage than the $\mathrm{H}$ I data, the $\mathrm{H} \alpha$ velocities here (from Amram et al. 1989) appear similar to the H I velocities; Amram et al. comment that the optical velocities along this arm remain almost constant at around $4075 \mathrm{~km} \mathrm{~s}^{-1}$. Models for ocular galaxies (e.g., Elmegreen et al. 1991; Paper II) generally predict nearly constant velocities along the tidal arms for these orientations, and Paper I finds that the ocular galaxy IC 2163, like NGC 2535, has constant velocity along much of the tidal tail.

The southern arm (the tidal bridge) does not have similar kinks in the velocity contours crossing the arm (see Fig. 8). The $\mathrm{H} \alpha$ velocity field in Amram et al. (1989) shows that after leaving the eye-shaped oval, the isovelocity contour at the systemic velocity turns and runs nearly parallel to the southern arm for the next $25^{\prime \prime}$. This behavior is also evident in the $\mathrm{H}$ I channel map at $v=4094 \mathrm{~km} \mathrm{~s}^{-1}$ in Fig. 10. Along the ridge line of the tidal bridge, the mean velocity is nearly constant at $4120 \mathrm{~km} \mathrm{~s}^{-1}$ in $\mathrm{H} \mathrm{I}$ (and $4125 \mathrm{~km} \mathrm{~s}^{-1}$ in $\mathrm{H} \alpha$ ) between the eye-shaped oval and the companion galaxy. The 

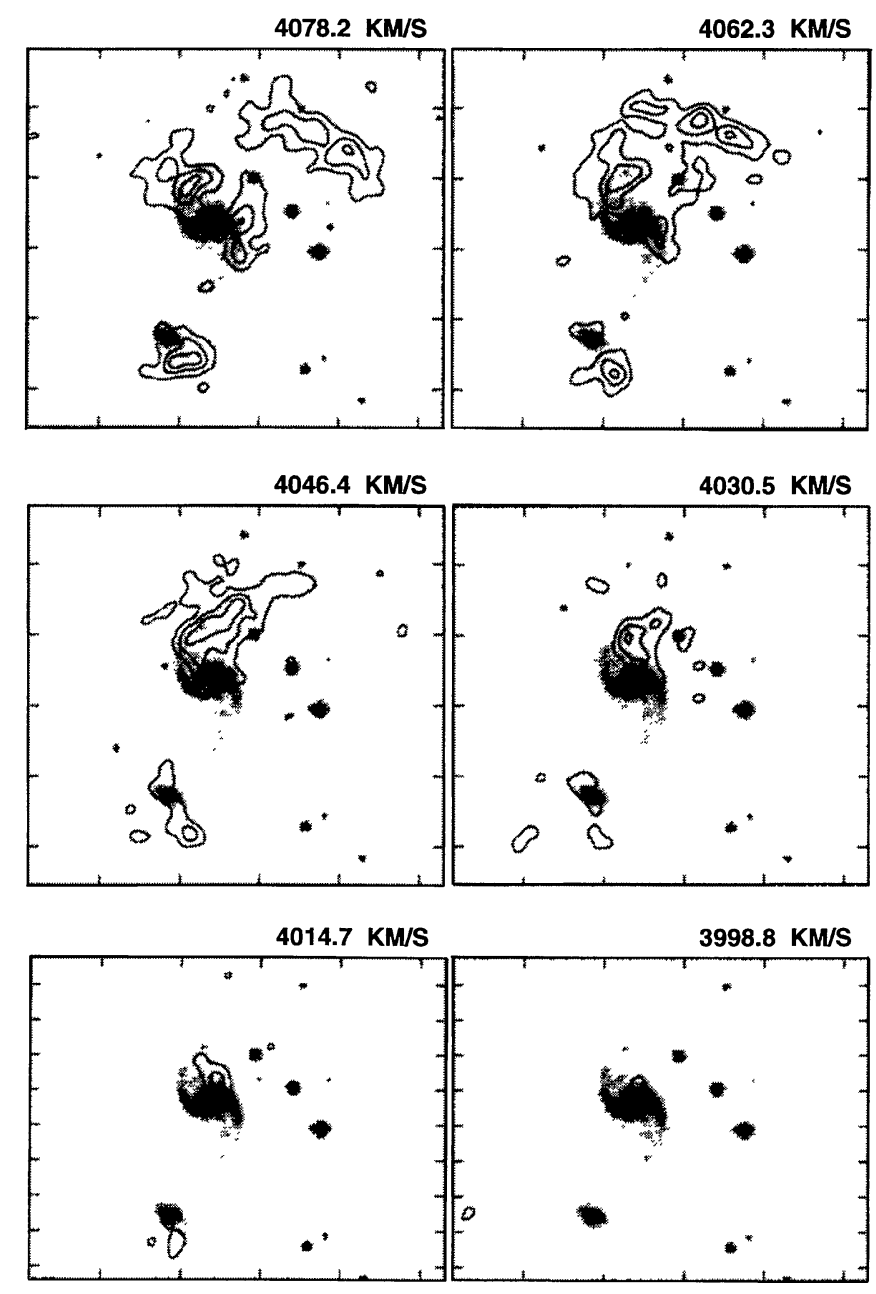

FIG. 10. (continued)

$\mathrm{H} \alpha$ velocity field exhibits a $60 \mathrm{~km} \mathrm{~s}^{-1}$ velocity difference across (i.e., perpendicular to) the broad tidal bridge. The above twist of the velocity contours and the constant velocity along (i.e., parallel to) much of the bridge resembles the velocity anomalies in the generic ocular model by Elmegreen et al. (1991).

The $\mathrm{H}$ I velocities also provide evidence for streaming motions around the eye-shaped oval. The highest velocity gas on the southern rim of the eye-shaped oval is displaced $22^{\circ} \pm 7^{\circ}$ clockwise from the adopted line of nodes of the projection at $\mathrm{PA}=153^{\circ}$ (notice the closed high-velocity contour near the eastern apex in the top panel of Fig. 9 and the location of the $\mathrm{H} \mathrm{I}$ emission in the $4189 \mathrm{~km} \mathrm{~s}^{-1}$ channel in Fig. 10). In the $\mathrm{H}$ I velocity field (which has a velocity resolution of $\left.5.3 \mathrm{~km} \mathrm{~s}^{-1}\right)$, the value of $\left(v_{\mathrm{obs}}-v_{\mathrm{sys}}\right)$ is $7-8 \mathrm{~km} \mathrm{~s}^{-1}$ greater at $\mathrm{PA}=131^{\circ}$ on the eye-shaped oval than on the southeastern line-of-nodes $\left(\mathrm{PA}=153^{\circ}\right)$ at the same radius. We rely on the $\mathrm{H}$ I velocity data for this small velocity difference because the $\mathrm{H} \alpha$ velocity data in Amram et al. (1989) have a spectral sampling of $19 \mathrm{~km} \mathrm{~s}^{-1}$. If the motion is confined to the plane of the disk, then

$$
\left(v_{\mathrm{obs}}-v_{\mathrm{sys}}\right) / \sin i=v_{c} \cos \theta+\Delta v_{t} \cos \theta+v_{r} \sin \theta,
$$

where $v_{c}$ is the circular velocity, $\Delta v_{t}$ is the excess tangential velocity, $v_{r}$ is the radial velocity, and $\theta$ is the azimuthal angle measured counterclockwise in the face-on image. The displacement of $22^{\circ}$ in the sky-plane image corresponds to $\theta=-24^{\circ}$. For pure circular rotation, the value of ( $v_{\text {obs }}$ $\left.-v_{\text {sys }}\right)$ should be $4 \%$ to $13 \%$ lower at $\theta=-24^{\circ} \pm 7^{\circ}$ than on the line of nodes, whereas the observed value in the $\mathrm{H} \mathrm{I}$ velocity field is $16 \%-18 \%$ higher than on the line of nodes. After deprojection with $i=23^{\circ} \pm 4^{\circ}$, the observed excess velocity at $\mathrm{PA}=131^{\circ}$ relative to that on the line of nodes is $29 \pm 9 \mathrm{~km} \mathrm{~s}^{-1}$. Thus, there are noncircular motions in this part of NGC 2535. Paper I finds similar evidence for streaming motions along the eye-shaped oval in IC 2163.

Because of the noncircular motions on the tidal arms and around the eye-shaped oval, a slice of the $\mathrm{HI}$ velocity field through the nucleus of NGC 2535 along the line of nodes of the projection should not be regarded as the rotation curve.

\subsubsection{Outer envelope of $N G C 2535$}

Notice from Fig. 8 that the velocity at the western end of the tidal tail is $4130 \mathrm{~km} \mathrm{~s}^{-1}$, about the same as the velocity just south of the eye-shaped oval, and the velocity at the 


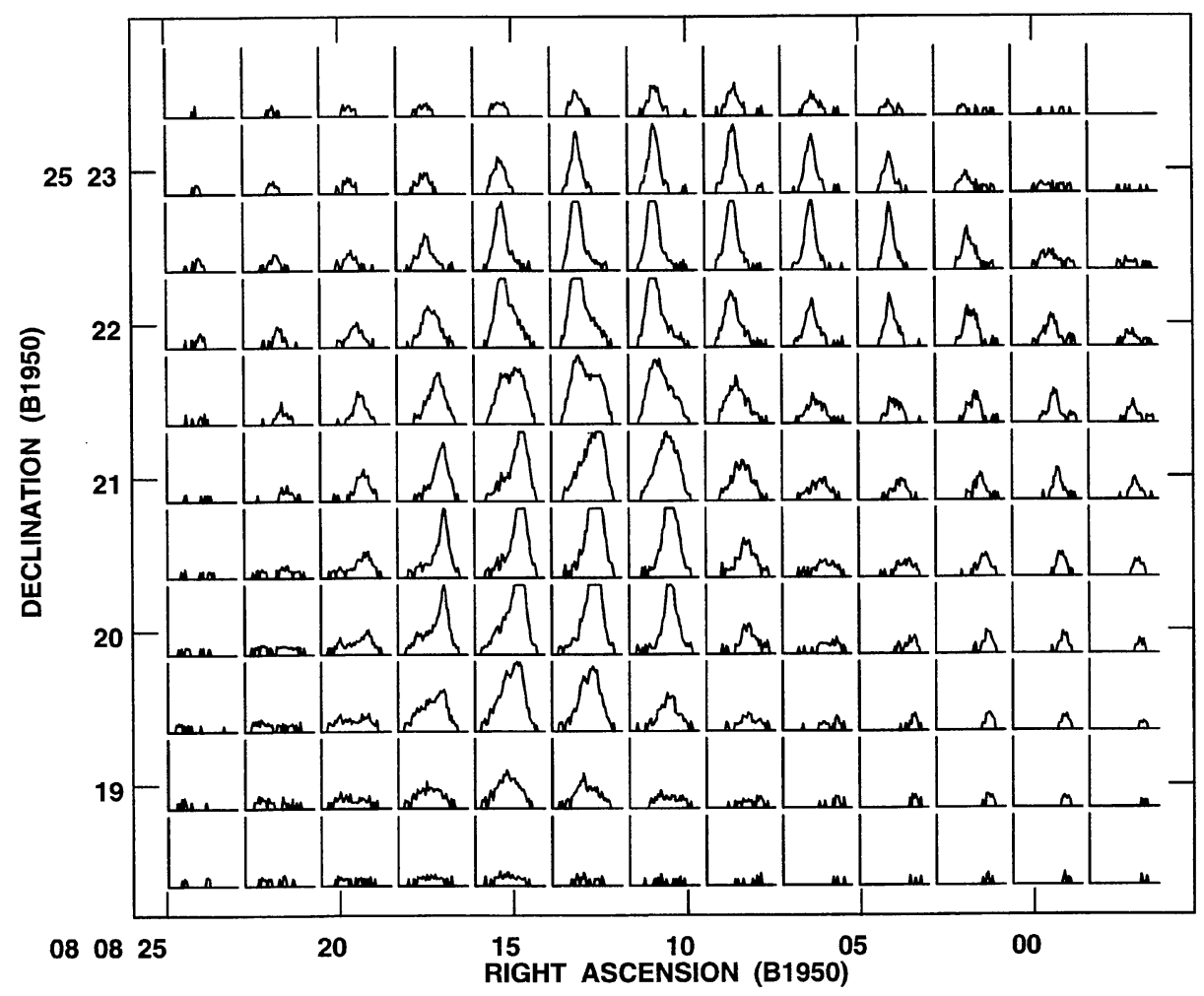

FIG. 11. $\mathrm{H}$ I line profiles from a cube convolved to $60^{\prime \prime}$ resolution to have greater sensitivity to the emission from the $\mathrm{H}$ I envelope. The velocity scale on the abscissa goes left to right from 3956 to $4221 \mathrm{~km} \mathrm{~s}^{-1}$, and the surface brightness scale on the ordinate goes from 0 to $30 \mathrm{mJy}^{-1}$ beam ${ }^{-1}$. The rms noise is $1.4 \mathrm{mJy}^{-1}$. The field displayed is approximately the same as in Fig. 1.

southern end of the tidal bridge is $4060 \mathrm{~km} \mathrm{~s}^{-1}$, about the same as the velocity just north of the eye-shaped oval. The perturbations in velocity at the outer ends of the tidal tail and the tidal bridge are opposite in sign and have about the same magnitude relative to the systemic velocity but do not occur at diametrically-opposite positions. The global $\mathrm{H}$ I velocity field (bottom panel of Fig. 9) shows that the trend of increasing velocity along the tidal tail as one goes from the northwest to the west continues in the $\mathrm{HI}$ envelope around to the southwest to $v=4150 \mathrm{~km} \mathrm{~s}^{-1}$. The velocity field in the west and southwest appears to be dominated by tidal streaming due to the interaction and is not clearly related to the velocity field of the central disk. Figure 11 presents line profiles from an $\mathrm{H}$ I cube convolved to $60^{\prime \prime}$ resolution to have greater sensitivity to the emission from the envelope. Most of the line profiles in the envelope have a broad velocity component. For example, in the southwestern envelope the profile in the 4th column from the right, 5th row from the bottom in Fig. 11 has FWHM $=82 \mathrm{~km} \mathrm{~s}^{-1}$. Paper I found that NGC 2207, the companion to the ocular galaxy IC 2163, has a large pool of gas extending $40 \mathrm{kpc}$ south of the main disk; however, unlike the envelope of NGC 2535, the line profiles in the southern extension of NGC 2207 are relatively narrow. Apparently, in NGC 2535, the gas in the envelope is still in orbits that have been highly disturbed by the interaction.

The high values of the velocity near the end of the tidal tail and in the western to southwestern part of the envelope require a peculiar velocity component of $40-60 \mathrm{~km} \mathrm{~s}^{-1}$ away from us on what is mainly the approaching side of NGC 2535. This is a very recent encounter, the gas in the envelope is still stirred up, as evidenced by the high velocity dispersion, and we are viewing the central disk close to faceon. Therefore, we suspect that these peculiar velocities are mostly $z$ motions that may eventually lead to the outer part of the disk bending away from us here. A nearly in-plane encounter should not produce a strong warp at the present stage.

In the velocity field in the extreme southern part of the system (see Fig. 8), we are seeing the result of strong tidal accelerations in NGC 2535 produced by the proximity of the companion plus the superposition of the velocity field of NGC 2536. In Klaric's model, the tidal bridge gas here is now mainly below the initial disk of NGC 2535, NGC 2536 is just below the initial disk plane of NGC 2535, and thus NGC 2636 is on the near side (relative to us) of most of the tidal bridge gas. This geometry is consistent with the blue color of NGC 2536. In the model, bridge material is being rapidly accreted by NGC 2536 , which means that this material has a peculiar velocity component headed towards us. This is consistent with the observed sense of the velocity perturbation.

Amram et al. (1989) previously invoked a warp of the disk of NGC 2535 to account for the apparent steep drop in the $\mathrm{H} \alpha$ "rotation" velocity beyond the eye-shaped oval. We agree with them that their $\mathrm{H} \alpha$ "rotation curve" does not represent the true rotation of the galaxy. Velocity streaming, 

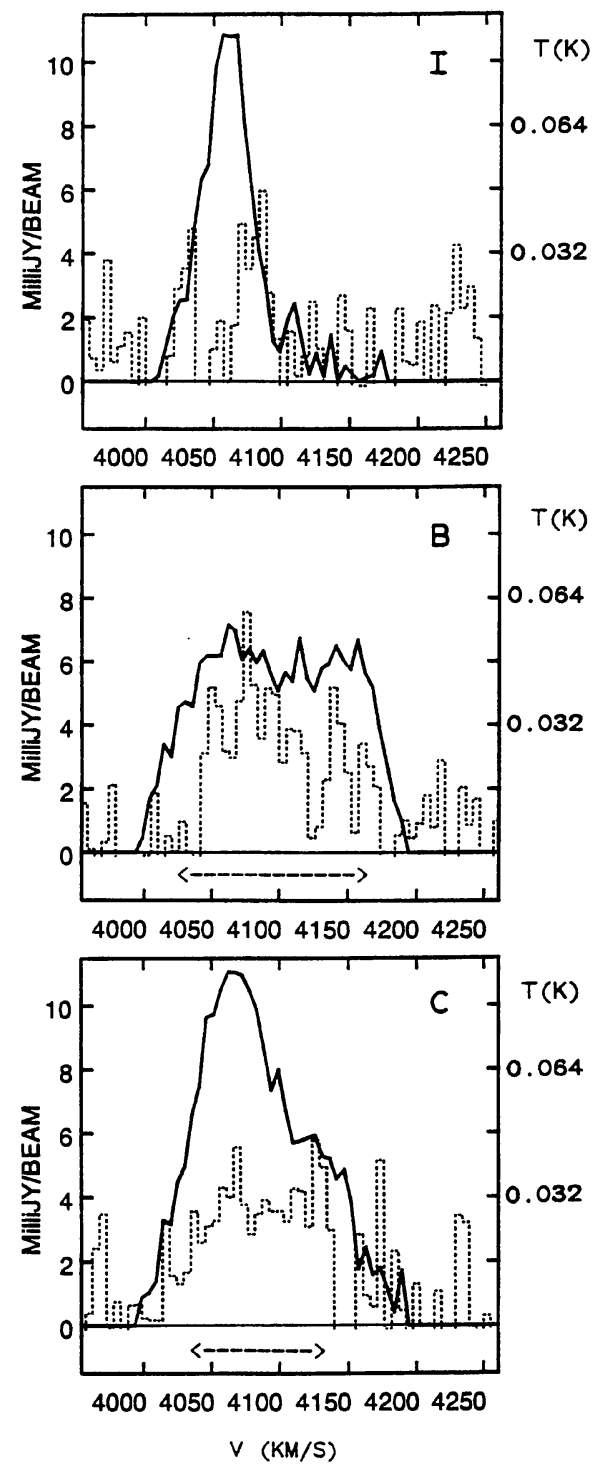

FIG. 12. A comparison of $\mathrm{H}$ I line profiles (solid curve) and $\mathrm{CO}$ line profiles (dashed curve) at ${ }^{12} \mathrm{CO}$ positions $\mathrm{B}, \mathrm{C}$, and I. The $\mathrm{H}$ I line profiles are from an $\mathrm{HI}$ cube convolved to the same $33^{\prime \prime}$ resolution as the $\mathrm{CO}$ data. The $\mathrm{H} \mathrm{I}$ intensity scale is marked on the left in mJy beam ${ }^{-1}$, and the $T_{\mathrm{mb}}$ scale for the $\mathrm{CO}$ emission is marked on the right in kelvin. The rms noise in the convolved $\mathrm{H}$ I cube is $0.90 \mathrm{mJy}^{\text {beam }}{ }^{-1}$. The bar below the axis at positions $\mathrm{B}$ and $\mathrm{C}$ indicates the range of the $\mathrm{H} \alpha$ velocity field (from Amram et al. 1989).

as discussed in the previous section, seems to account for the velocity behavior that they attributed to the effect of a warp within the optical disk (i.e., out to $R_{25}$ ); the $\mathrm{H}$ I velocity field, which has better coverage than the $\mathrm{H} \alpha$ velocity field, shows no evidence of a warp within the optical disk. It is well beyond the optical disk that we detect evidence for $z$ motions in the H I velocity field.

\subsection{Comparison of $\mathrm{HI}, \mathrm{CO}$, and $\mathrm{H \alpha}$ Velocities}

The $\mathrm{H} \alpha$ velocity field gives the same values, within the uncertainties, as the $\mathrm{H}$ I velocity field for the systemic velocity of NGC 2535 and for the nearly constant velocities along much of the tidal arms of NGC 2535, but there are some differences at the rim of the eye-shaped oval. For example, at a position angle of $-30^{\circ}$ to $-50^{\circ}$, the $\mathrm{H} \alpha$ mean velocity on the northwestern rim of the eye-shaped oval is $4025 \mathrm{~km} \mathrm{~s}^{-1}$ (Amram et al.), whereas the $\mathrm{HI}$ mean velocity is 4050-4060 $\mathrm{km} \mathrm{s}^{-1}$. In the $\mathrm{H}$ I channel maps of Fig. 10, one sees that there is some $\mathrm{HI}$ here at the same velocity as the $\mathrm{H} \alpha$ emission but the dominant $\mathrm{H}$ I has higher velocity. Presumably, the $\mathrm{HI}-\mathrm{H} \alpha$ velocity difference results from the lower spatial resolution of the $\mathrm{H}$ I data.

In Fig. 12 we compare the ${ }^{12} \mathrm{CO}$ and $\mathrm{H}$ I line profiles after convolving the $\mathrm{H} \mathrm{I}$ cube to the same spatial resolution as the $\mathrm{CO}$ data. At all three positions, the $\mathrm{H} \mathrm{I}$ data have higher signal/noise than the $\mathrm{CO}$ data. For ${ }^{12} \mathrm{CO}$ positions $\mathrm{B}$ and $\mathrm{C}$ (the two clear $\mathrm{CO}$ detections), Fig. 12 indicates the range of the $\mathrm{H} \alpha$ velocity field at these locations (as read off the $\mathrm{H} \alpha$ velocity field figure in Amram et al. 1989); the total range of the $\mathrm{H} \alpha$ profile would be somewhat greater than this. For position I (the weak $\mathrm{CO}$ detection), no $\mathrm{H} \alpha$ velocity information is available.

At the central position (position $\mathrm{B}$ ), the $\mathrm{HI}$ profile is broad, flat, and fairly symmetric about the systemic velocity. The aperture includes the western apex but not the eastern apex of the eye-shaped oval. The ${ }^{12} \mathrm{CO}$ profile is less symmetric about the systemic velocity. $\mathrm{CO}$ emission is not detected at velocities $<4045 \mathrm{~km} \mathrm{~s}^{-1}$, corresponding to the northwestern rim of the eye-shaped oval. The strongest $\mathrm{CO}$ emission (4050-4120 $\mathrm{km} \mathrm{s}^{-1}$ ) includes velocities expected for the central region and for the western apex of the oval, where there is a very bright $\mathrm{HII}$ region with $\mathrm{H} \alpha$ velocity $=4065-4075 \mathrm{~km} \mathrm{~s}^{-1}$.

Position $\mathrm{C}$ includes part of the tidal tail just beyond the eastern apex of the eye-shaped oval, massive $\mathrm{H}$ I Cloud 2 (labelled in Fig. 6), and the optical spur (see Fig. 5). The CO velocity profile is rather broad and flat with FWHM $=119 \mathrm{~km} \mathrm{~s}^{-1}$. The $\mathrm{H} \mathrm{I}$ emission has the same range of velocities as the $\mathrm{CO}$ emission. However, unlike the $\mathrm{CO}$ profile, the $\mathrm{H}$ I profile is asymmetric with a strong maximum at $4036-4104 \mathrm{~km} \mathrm{~s}^{-1}$; inspection of the high resolution $\mathrm{H} \mathrm{I}$ cube confirms that this $\mathrm{H}$ I peak is from Cloud 2. Thus, at position $\mathrm{C}$ the atomic gas is more concentrated to Cloud 2 than the molecular gas, and much of the $\mathrm{CO}$ emission is not associated with Cloud 2.

At position I (which is on the elliptical arc), there is only a $10 \mathrm{~km} \mathrm{~s}^{-1}$ difference in the $\mathrm{H}$ I velocity field across the $\mathrm{CO}$ aperture. The $\mathrm{H} \mathrm{I}$ emission is centered at $4070 \mathrm{~km} \mathrm{~s}^{-1}$ with a FWHM linewidth of $47 \mathrm{~km} \mathrm{~s}^{-1}$; the CO emission, centered at $4081 \mathrm{~km} \mathrm{~s}^{-1}$ with a FWHM linewidth of $27 \mathrm{~km} \mathrm{~s}^{-1}$, comes from part of the same feature as the $\mathrm{H} \mathrm{I}$.

\subsection{H I Velocity Dispersion in NGC 2535}

The top panel of Fig. 13 presents the low resolution image of $\sigma_{v, g}$, the one-dimensional $\mathrm{HI}$ velocity dispersion, in greyscale overlaid with contours from the high resolution $N(\mathrm{H} \mathrm{I})$ image. The velocity dispersion $\sigma_{v, g}$ has been corrected for the velocity gradient across the beam but not for systematic velocity gradients along line of sight. These $\mathrm{H} \mathrm{I}$ velocity dispersions are high, $30 \mathrm{~km} \mathrm{~s}^{-1}$ over much of the disk, as compared to the values of $6-10 \mathrm{~km} \mathrm{~s}^{-1}$ (Dickey 


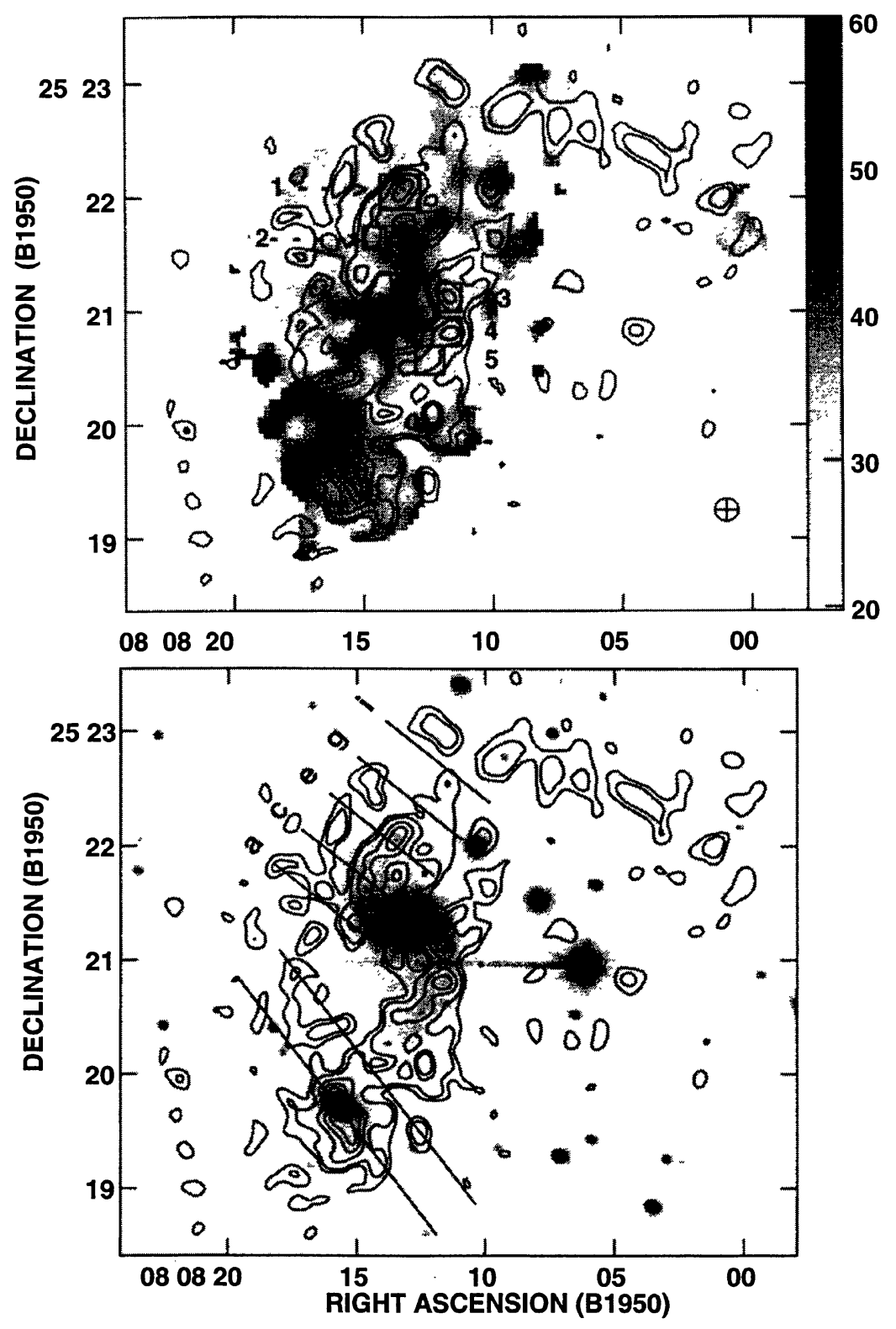

FIG. 13. Top: $\mathrm{H}$ I velocity dispersion in greyscale from the low resolution $\mathrm{H} \mathrm{I}$ image overlaid with the high resolution $N(\mathrm{H}$ I) contours from Fig. 5. The beam symbol represents the $N(\mathrm{H} \mathrm{I})$ resolution. The greyscale wedge runs from 20 to $60 \mathrm{~km} \mathrm{~s}^{-1}$. There is widespread high velocity dispersion in the $\mathrm{H}$ I gas as compared to undisturbed spirals. Numbered boxes are drawn around the five unusually massive $\mathrm{H}$ I clouds. Bottom: I-band image in greyscale overlaid with the same $N(\mathrm{H}$ I $)$ contours. There are no major clumps in the older stellar population at the locations of the massive $\mathrm{H}$ I clouds. The five slice-lines labeled a, $\mathrm{c}, \mathrm{e}, \mathrm{g}$, and i show the positions of the P-V diagrams thus labeled in Fig. 14. The two unlabeled slice-lines show the locations of the P-V diagrams of the tidal bridge and NGC 2536 in Fig. 15.

et al. 1990; van der Kruit \& Shostak 1984, and references therein) or $6-13 \mathrm{~km} \mathrm{~s}^{-1}$ (Kamphuis 1993) measured in undisturbed spirals. Thus, like the interacting pair IC 2163/ NGC 2207 and the interacting pair NGC 5774/75, NGC 2535 exhibits widespread, enhanced $\mathrm{H}$ I velocity dispersion. Amram et al. (1989) note that there is also a large dispersion in the $\mathrm{H} \alpha$ velocities in NGC 2535. Some speculations as to what the measured high values for the velocity dispersion could represent are the following: we may be seeing enhanced turbulence resulting from collisions between tidally- accelerated clouds, or, within the beam, we may be seeing a collection of clouds differing in $z$ velocity as they stream perpendicular to the disk.

The $\mathrm{H}$ I velocity dispersion in NGC 2535 is comparable to the stellar velocity dispersion $\sigma_{v, s}$ in the $z$ direction in the solar neighborhood: for stars in the old thin disk, the local galactic $\sigma_{v, s}=20 \mathrm{~km} \mathrm{~s}^{-1}$ and the stellar scale height $H_{s}$ $=350 \mathrm{pc}$, and for stars in the thick disk, $\sigma_{v, s}=45 \mathrm{~km} \mathrm{~s}^{-1}$ and $H_{s}=1 \mathrm{kpc}$ (Freeman 1987). In the case of an isothermal disk in hydrostatic equilibrium, the $\mathrm{H}$ I scale height in the 
TABLE 5. Massive $\mathrm{H}$ I clouds in NGC 2535.

\begin{tabular}{ccccccccc}
\hline \hline Cloud & $\alpha(1950)$ & $\delta(1950)$ & $\begin{array}{c}\mathrm{N}(\mathrm{HI}) \\
\left(\mathrm{M}_{\odot} \mathrm{pc}^{-2}\right)\end{array}$ & $\begin{array}{c}\sigma_{\mathrm{v}}(\mathrm{HI}) \\
\left(\mathrm{km} \mathrm{s}^{-1}\right)\end{array}$ & $\begin{array}{c}\mathrm{M}(\mathrm{HI}) \\
\left(10^{8} \mathrm{M}_{\odot}\right)\end{array}$ & $\begin{array}{c}a \\
(\mathrm{kpc})\end{array}$ & $\begin{array}{c}\mathrm{M}_{\mathrm{vir}} \\
\left(10^{8} \mathrm{M}_{\odot}\right)\end{array}$ & $\begin{array}{c}\mathrm{L}_{B} / \mathrm{pc}^{2} \\
\left(\mathrm{~L}_{\left.B \odot \mathrm{pc}^{-2}\right)}\right.\end{array}$ \\
\hline 1 & 080813.5 & 252206 & 10 & 33 & 3.0 & 2.8 & 25 & 23 \\
2 & 080814.8 & 252139 & 10 & 35 & 1.4 & 1.5 & 15 & 57 \\
3 & 080811.7 & 252109 & 12 & 31 & 1.8 & 1.8 & 14 & 75 \\
4 & 080811.7 & 252048 & 11 & 30 & 2.2 & 2.0 & 15 & 45 \\
5 & 080812.6 & 252033 & 10 & 29 & 1.9 & 2.0 & 14 & 35 \\
\hline \hline
\end{tabular}

main disk, where the stellar potential dominates the $Z$ force, is

$$
H_{g} \approx \frac{\sigma_{v, g} \sigma_{v, s}}{\pi G \mu}
$$

where $\mu$ is the total mass surface density (van der Kruit 1989), and the stellar scale height

$$
H_{s} \approx \frac{\left[\sigma_{v, s}\right]^{2}}{\pi G \mu} .
$$

For the case where the mass surface density of stars $\mu_{s}$ does not necessarily dominate the mass surface density of gas $\mu_{g}$, an expression for the $\mathrm{H}$ I scale height can be derived from the approximate equation in Elmegreen (1989) for the central disk pressure,

$$
P(0)=\rho_{g}(0)\left[\sigma_{v, g}\right]^{2} \approx\left(\pi G \mu_{g} / 2\right)\left[\mu_{g}+\mu_{s}\left(\sigma_{v, g} / \sigma_{v, s}\right)\right] .
$$

Thus the H I scale height is given by

$$
H_{g}=\frac{\mu_{g}}{2 \rho_{g}(0)} \approx \frac{\left[\sigma_{v, g}\right]^{2}}{\pi G\left[\mu_{g}+\mu_{s} \sigma_{v, g} / \sigma_{v, s}\right]} .
$$

This reduces to Eq. (2) if $\mu_{g}$ is small. For the same tidal acceleration, the percentage increase in the velocity dispersion of the gas is greater than that of the stars because the gas has the smaller initial velocity dispersion. Also, for the type of encounter that produces ocular structure, the main tidal acceleration is in-plane, and the disturbed velocities in the stars will take longer to isotropize than those in the gas, since the stars form a collisionless system. Many of the $\mathrm{H}$ I line profiles of NGC 2535 are asymmetric; this was also the situation in NGC 2207 (see Paper I). Whether or not the assumption of an isothermal gas disk in hydrostatic equilibrium is appropriate for these very recently disturbed galaxies, the $\mathrm{H}$ I gas in NGC 2535 may well have a large scale height as a consequence of the enhanced turbulence and possible tidal perturbations in the $z$-direction (evidence for the latter was presented in the discussion of the outer envelope in Sec. 5.2.3). In the case of the interacting galaxy NGC 5775 (which is viewed close to edge-on), Irwin (1994) fit a galaxy model to the complete set of $\mathrm{H}$ I line profiles and found that the best fit occurred with a thick exponential disk with $H_{g}$ $=1.1 \mathrm{kpc}$ and $\sigma_{v, g}=24 \mathrm{~km} \mathrm{~s}^{-1}$. Thus we should allow for the possibility that the $\mathrm{H}$ I scale height may include most of the stars.

\subsection{Massive H I Clouds in NGC 2535}

In the bottom panel of Fig. 6 and the top panel of Fig. 13, boxes are drawn around five $\mathrm{H}$ I clouds, each with $\mathrm{H}$ I mass in excess of $10^{8} \mathscr{C}_{\odot}$. Table 5 lists the following properties of these unusually massive $\mathrm{H}$ I clouds on the tidal arms of NGC 2535: the position, the average $\mathrm{H}$ I column density $N(\mathrm{H} \mathrm{I})$ inside the box, the average one-dimensional $\mathrm{H}$ I velocity dispersion $\sigma_{v, g}$, the total $\mathrm{H}$ I mass $M(\mathrm{H} \mathrm{I})$ in the box, the cloud semi-major axis $a$, the virial mass $M_{\text {vir }}$, and the average $B$-band surface brightness $L_{B} / \mathrm{pc}^{2}$ before deprojection. The values of $\sigma_{v, g}$ in this table have been corrected for the velocity gradient across the synthesized beam but not for systematic velocity gradients along the line of sight. The rms scatter in the values of $\sigma_{v, g}$ within each cloud is $\pm 4 \mathrm{~km} \mathrm{~s}^{-1}$. The calibration of our $B$ image is uncertain by $\pm 0.25 \mathrm{mag}$. The values of $L_{B}$ have not been corrected for extinction. The $B$-band extinction $A_{B}$ would be $0.4 \mathrm{mag}$ if half of $N(\mathrm{H} \mathrm{I})$ were in front of all the stars, but less if the stars and the $\mathrm{H} I$ gas have similar scale heights.

The virial mass of a gas cloud is

$$
\mathscr{M}_{\mathrm{vir}}=\frac{C \sigma_{v}^{2} a}{G},
$$

where for a sphere of uniform density, $C=5$, and for an oblate spheroid of uniform density and eccentricity $e, C$ $=5 e /(\arcsin e)$ (from the expression for the gravitational potential energy of an oblate spheroid in Chandrasekar 1969 and in Binney \& Tremaine 1987). The value of $C$ ranges from 5 for a sphere to 3.18 in the limit of a very thin spheroid in which the minor axis $\rightarrow 0$; with a minor/major axis ratio of 0.4 , the value of $C$ is only $25 \%$ greater than when the minor axis $\rightarrow 0$. As the virial mass is not very sensitive to the axis ratio when the axis ratio is less than 0.4 , we computed the values of $\mathscr{C l}_{\text {vir }}$ in Table 5 for an oblate spheroid with uniform density and minor/major axis ratio $=0.15$; then $C$ $=3.48$. Since the average value of the cloud semimajor axis is $2 \mathrm{kpc}$ (see Table 5), the adopted axis ratio corresponds to a semi-minor axis of $0.3 \mathrm{kpc}$, which is a conservatively low estimate. For the semi-major axis $a$, we took $(A / \pi)^{1 / 2}$, where $A$ is the plane-of-sky area of the box in the high resolution $N(\mathrm{H} \mathrm{I})$ image, then deconvolved from the synthesized beam, and multiplied by $(\cos i)^{-1 / 2}=1.04$ to correct to faceon. In the case of Cloud 1, we were able to fit a 2D Gaussian to the distribution of $\mathrm{H} \mathrm{I}$ emission; this gave a geometric mean, deconvolved radius of $2.1 \mathrm{kpc}$, instead of the $2.8 \mathrm{kpc}$ value from the box area. This suggests that computing the value of $a$ from the box area may lead to, perhaps, a $30 \%$ overestimate of $a$ and $M_{\text {vir }}$, as Cloud 1 is the worst example of fitting a box to the $N(\mathrm{H} \mathrm{I})$ contours.

These massive $\mathrm{H}$ I clouds do not coincide with the most luminous blue knots in NGC 2535, and Cloud 1 is the only massive $\mathrm{H}$ I cloud that appears to contain an unusually bright 
clump of star formation (see Fig. 6 and the $\mathrm{H} \alpha$ image in Amram et al. 1989). From an $N$-body/SPH simulation of galaxy encounters, Barnes \& Hernquist (1992) concluded that massive clumps on tidal arms form from instabilities in the stellar system, with the gas collecting subsequently in the stellar potential well. The $I$-band image in the bottom panel of Fig. 13 shows that the five massive H I clouds in NGC 2535 do not correspond in general to major clumps in the older stellar population. Cloud 1 has some excess $I$-band emission, but this may be from young stars, since new star formation has already started here. In Table 5 , the average value of $L_{B} / \mathrm{pc}^{2}$ for Cloud 1 is less than for the other clouds because Cloud 1 includes the optical interarm region.

The virial masses of these clouds from Eq. (6) are a factor of 7-11 times the observed $M(\mathrm{HI})$ because the values of $\sigma_{v, g}$ are large. In at least four of the five clouds, our upper limits to the molecular mass are insufficient to account for the difference. Only Cloud 2 lies in a field where ${ }^{12} \mathrm{CO}$ emission is clearly detected $\left({ }^{12} \mathrm{CO}\right.$ position $\left.\mathrm{C}\right)$. As noted in Sec. 5.3 above, much of the $\mathrm{CO}$ emission at position $\mathrm{C}$ is probably not from Cloud 2. Nevertheless, if all the ${ }^{12} \mathrm{CO}$ emission from position $\mathrm{C}$ were associated with Cloud 2, then this cloud would have a total mass in gas (including a $40 \%$ by mass contribution from helium) of $1.5 \times 10^{9} \mathscr{C}_{\odot}$, which is about the same as $M_{\mathrm{vir}}$. At the positions of the other clouds, our $2 \times$ the rms noise upper limits on the molecular mass are comparable to the measured $\mathrm{H}$ I mass.

Various possible explanations for the discrepancy between the virial mass and the gas mass of these clouds are (1) the $\mathrm{H} \mathrm{I}$ scale height is comparable to the stellar scale height and thus the stellar mass $\mathscr{C l}_{s}$ inside the cloud is much larger than the gas mass $\mathscr{C}_{\mathrm{g}}$ [see the discussion in Sec. 5.4 and Eq. (7) below], (2) the clouds are not virialized (perhaps they are still in the process of forming), or (3) there is a significant contribution to the measured $\mathrm{HI}$ velocity dispersions from velocities other than turbulence. Although the stars are not clumped like the gas, the values of the $B$-band surface brightness in Table 5 imply that the stellar surface density at these locations is large compared to the gas surface density. If we take $\mathscr{C} / L=3$ in $B$ band from Binney \& Tremaine (1987) for visible stars in the solar neighborhood, then the ratio of stellar surface density to $\mathrm{H}$ I surface density varies from 7 for Cloud 1 to 19 for Cloud 3, and thus, the stellar mass could account for the difference between the virial mass and the gas mass. That is, $\mathscr{C}_{\text {vir }}=\mathscr{C}_{g}+\mathscr{M}_{s}$ satisfies Eq. (6) for $\sigma_{v}$ equal to the observed $\mathrm{H}$ I velocity dispersion. This situation is unusual for interstellar clouds because usually $\sigma_{v, g} \ll \sigma_{v, s}$. A version of the virial equation that includes both stars and gas can be obtained by multiplying Eq. (5) for the gas scale height $H_{g}$ by the cloud area $A_{0}$ in the plane to get

$$
\mathscr{M}_{g}+\left(\sigma_{v, g} / \sigma_{v, s}\right) \mathscr{C}_{\mathrm{stars}, T} \approx \frac{\sigma_{v, g}^{2} A_{0}}{\pi G H_{g}}
$$

where $\mathscr{C}_{\text {stars, } T}=\mu_{s} A_{0}$ is the total stellar mass in the column through the disk, not just the stellar mass $\mathscr{L}_{s}$ inside the cloud, and $\mathscr{M}_{g}=\mu_{g} A_{0}$. Thus when $\sigma_{v, g}$ is comparable to $\sigma_{v, s}$, the contribution of the stellar mass to the virial mass is significant. With $A_{0} / H_{g} \propto \pi a$, we return to the previous gas virial equation in the limit of $\sigma_{v, g} / \sigma_{v, s} \ll 1$.

In NGC 2535, we find (a) high $\mathrm{H}$ I velocity dispersion throughout much of the disk and (b) clouds comparable in mass to H I-rich dwarf galaxies but, generally, without major stellar clumps at the same location. Since a similar phenomenon has been found previously in other interacting pairs, IC 2163/NGC 2207 (Elmegreen et al. 1993 and Paper I) and NGC 5774/75 (Irwin 1994), enhanced turbulence and unusually massive gas clouds produced by gravitational instabilities in the gaseous disk may be a general feature of interacting spiral galaxies during a certain early phase of postencounter evolution.

\subsection{More About the Elliptical Arc and the Tidal Tail}

In the lower panel of Fig. 5, the ${ }^{12} \mathrm{CO}$ positions are marked on a display of the high resolution $N(\mathrm{H} \mathrm{I})$ image. Positions $\mathrm{C}$ (a clear detection) and $\mathrm{E}$ (a possible detection) are placed directly on the tidal tail seen in $\mathrm{H} \mathrm{I}$, whereas positions I (a weak detection) and $\mathrm{H}$ are on the part of the $\mathrm{HI}$ elliptical arc northeast of the tidal tail. To find where any molecular gas in this region is located, we also observed two positions, $\mathrm{CH}$ and EI between the tidal tail and the elliptical arc. Position $\mathrm{H}$ shows no detection, and the features in the line profiles at positions $\mathrm{CH}$ and $\mathrm{EI}$ are below $2 \times$ the rms noise and may result from overlap with positions $\mathrm{C}$ and $\mathrm{I}$. We thus exclude the possibility that the molecular gas is situated mainly between the H I features. The molecular gas in this region coincides either with the tidal tail (positions $\mathrm{C}$ and possibly E) or with the elliptical arc (position I). We conclude that the tidal tail and the elliptical arc are distinct features in the total gas. The origin of the elliptical arc will be considered in Sec. 8 .

To study the portions of the tidal tail and the elliptical arc that are parallel in projection, we made position-velocity diagrams of the $\mathrm{H} \mathrm{I}$ emission in this region. The P-V diagrams in panels a, c, e, g, and i of Fig. 14 were made at the locations of the five slice lines thus labeled in Fig. 13; the $\mathrm{P}-\mathrm{V}$ diagrams in panels $\mathrm{b}, \mathrm{d}, \mathrm{f}$, and $\mathrm{h}$ are along parallel slices midway between these. The ordinates in Fig. 14 are along the slice lines with positive displacements on the northeastern side. The P-V diagrams show similar velocities on the tidal tail and the elliptical arc. In many cases, these two features differ by less than the $5 \mathrm{~km} \mathrm{~s}^{-1}$ channel width; the greatest difference is $20 \mathrm{~km} \mathrm{~s}^{-1}$, and $\left(v_{\text {obs }}-v_{\text {sys }}\right)$ tends to be slightly more negative on the tail than on the elliptical arc. The low resolution $\mathrm{H}$ I cube shows that some of the emission between the elliptical arc and the tail has the same velocity as that on the elliptical arc.

In IC 2163, Paper I finds a double-parallel arm structure (tidal tail plus streaming arm) on the anti-companion side with a line-of-sight velocity difference of $70-100 \mathrm{~km} \mathrm{~s}^{-1}$ between the two parallel components of the tidal tail, as predicted by ocular models. If this feature had been spatially unresolved in IC 2163, its signature in a sequence of P-V diagrams would have been two peaks on the tidal tail well separated in velocity in each $\mathrm{P}-\mathrm{V}$ diagram but getting closer in velocity as one goes through the sequence. In NGC 2535 we do not detect the double-parallel arm morphology on the 

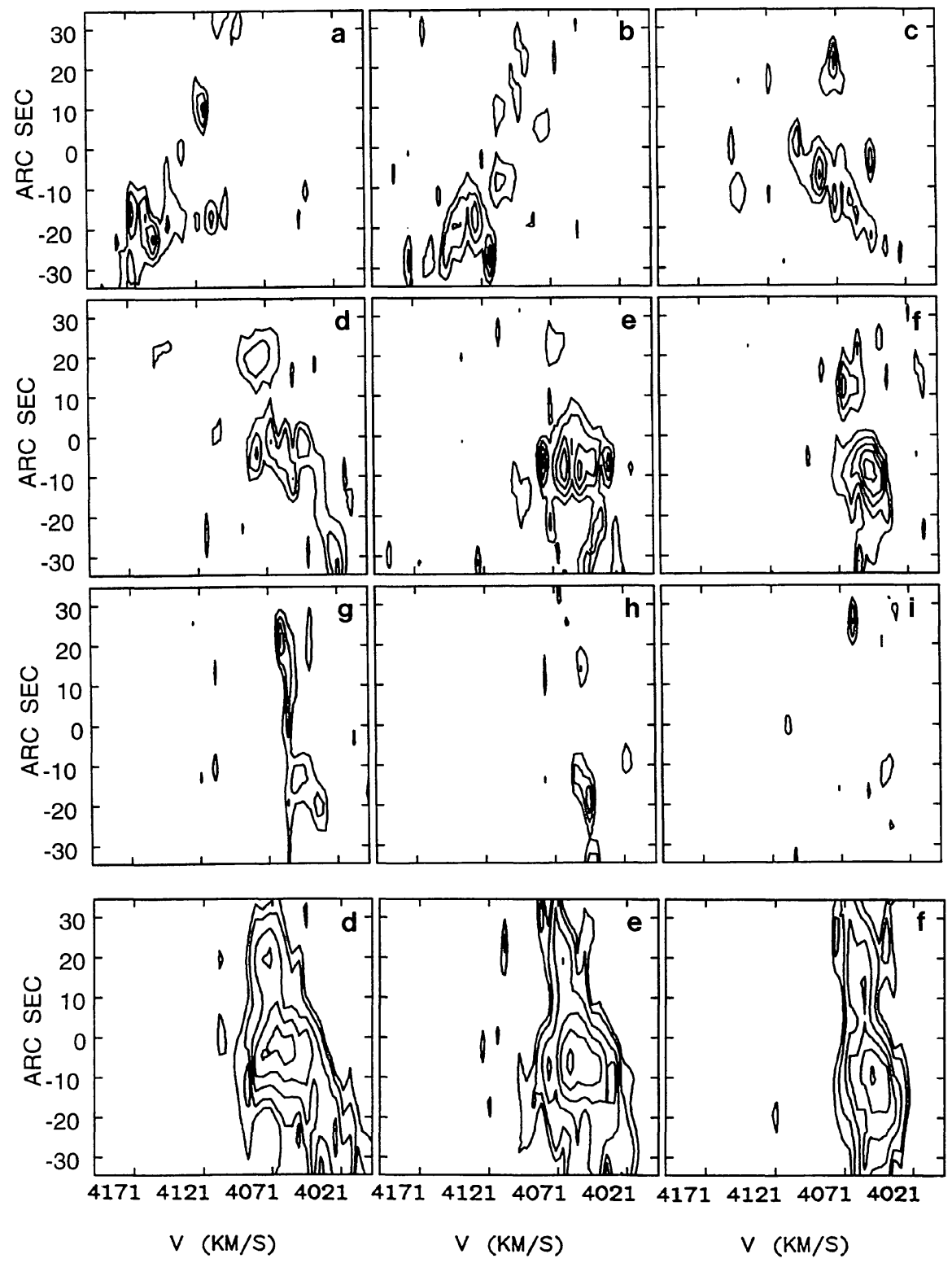

FIG. 14. Position-velocity diagrams of the $\mathrm{H}$ I emission from the tidal tail and the elliptical arc. The $\mathrm{P}-\mathrm{V}$ diagrams in panels a, $\mathrm{c}, \mathrm{e}, \mathrm{g}$, and $\mathrm{i}$ were made at the locations of the five slice-lines thus labeled in Fig. 13; the P-V diagrams in panels b, d, f, and $\mathrm{h}$ are along parallel slices midway between these. The ordinates are along the slice lines with displacements positive to the northeast and negative to the southwest. The top three rows are from the high resolution cube, with contour levels of $2,3,4,5$, and $6 \times$ the rms noise, and the lowest contour level is at $1.8 \mathrm{mJy}_{\text {beam }}^{-1}$, or $7.6 \mathrm{~K}$. The bottom row shows the same positions as the second row from the top, but from the low resolution cube with contour levels at $3,4,6,8,10$, and $12 \times$ the rms noise, and the lowest contour level is at $2.0 \mathrm{mJy}_{\text {beam }}{ }^{-1}$, or $4.6 \mathrm{~K}$. The tidal tail is the feature at $-20^{\prime \prime}$ to $+5^{\prime \prime}$, and the elliptical arc is the feature at $+5^{\prime \prime}$ to $+30^{\prime \prime}$.

tidal tail either in the optical image or in the P-V diagrams of Fig. 14. Notice that the tidal tail in Fig. 14 is more complicated than a double-parallel arm: for example, in each of panels $\mathrm{c}, \mathrm{d}$, and e, there appear to be 3 or 4 clumps on the tidal tail (covering a range of up to $50 \mathrm{~km} \mathrm{~s}^{-1}$ ) within the $3.0 \times 2.9 \mathrm{kpc}$ FWHM synthesized beam of the high resolution cube. This large range of velocities would be unusual in an undisturbed galaxy because the gradient in the velocity field is small along the tidal tail here (see Fig. 8). If these clumps at different velocities are all in the plane of NGC 2535 , then collisions between them should lead to enhanced turbulence. Another possibility is that they are not all in the plane of NGC 2535 and may differ in $z$ velocity. In Klarić's (1993) model for NGC 2535, the double-arm structure appears at somewhat earlier epoch, and the two components have now merged. The observed $\mathrm{P}-\mathrm{V}$ diagrams are consistent with the notion that we are viewing the double-arm structure after the two components mix.

\section{$5.7 N G C 2536$}

In this section we consider the various velocity components of the $\mathrm{HI}$ emission in the direction of NGC 2536. The end of the tidal bridge of NGC 2535 overlaps NGC 2536 


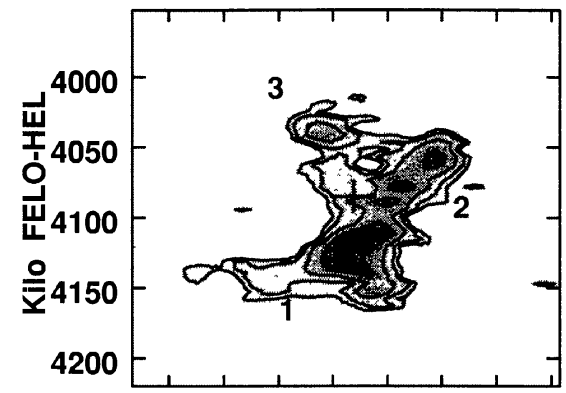

$\begin{array}{lllllllllll}08 & 08 & 19 & 18 & 17 & 16 & 15 & 14 & 13 & 12\end{array}$ RIGHT ASCENSION (B1950)

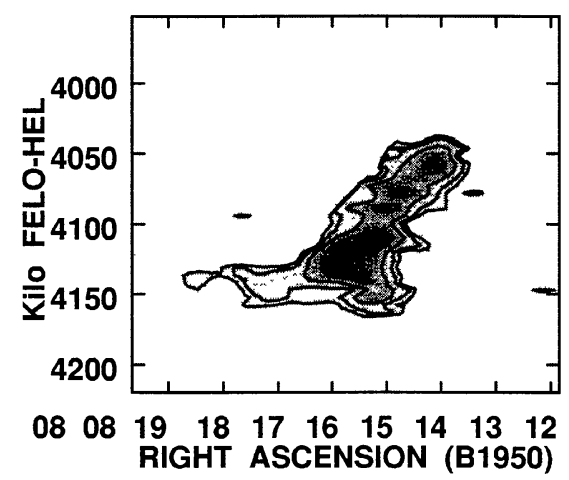

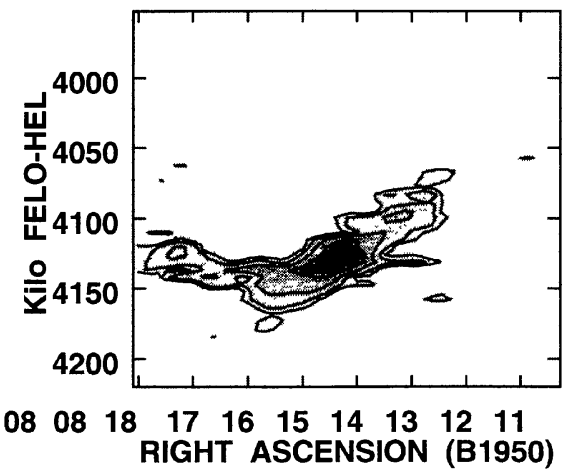

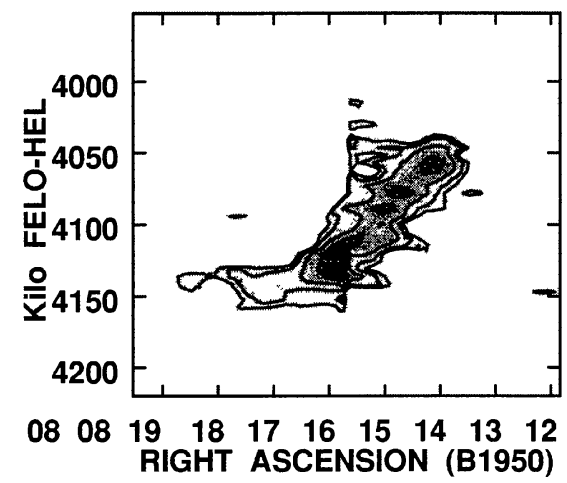

FIG. 15. H I position-velocity diagrams from three different cubes to show our attempts at separating the $\mathrm{H}$ I contributions of NGC 2536 and the tidal bridge of NGC 2535. The ordinate is the heliocentric velocity in $\mathrm{km} \mathrm{s}^{-1}$. For the upper left and the two lower panels, the abscissa is along the slice line through the optical nucleus of NGC 2536 in Fig. 13 at the position angle of the photometric major axis of NGC 2536. The upper right panel is a P-V diagram at the location of the parallel slice-line cutting across the bridge north of NGC 2536 in the same figure. The northeastern side is on the left in each P-V diagram. The upper left panel displays the combined emission from the bridge and NGC 2536 and is centered on the optical nucleus of NGC 2536 , marked by a plus sign. The lower two panels show the residual emission in the same field after two different attempts at removing the $\mathrm{H}$ I associated with NGC 2536 , as described in the text. The rms noise in these low resolution (naturally-weighted) images is $0.65 \mathrm{mJy} \mathrm{beam}^{-1}$, and the contour levels are at 3 , 4,6 , 9 , and 12 times the rms noise.

along line of sight in a clump with a combined $\mathrm{H}$ I mass of $2 \times 10^{9} \mathscr{C}_{\odot}$ and a peak $N\left(\mathrm{H}\right.$ I) of $21 \mathscr{M b}_{\odot} \mathrm{pc}^{-2}$. The location of maximum $N(\mathrm{H} \mathrm{I})$ is offset $8^{\prime \prime} \mathrm{SW}$ of the maximum surface brightness on the $I$-band and $B$-band images. The mean velocity of the $\mathrm{H} \mathrm{I}$ emission along most of the tidal bridge is $4120 \mathrm{~km} \mathrm{~s}^{-1}$, with velocity dispersions of $25-30 \mathrm{~km} \mathrm{~s}^{-1}$.

The complexity of the situation is illustrated by the position-velocity diagrams of the tidal bridge and NGC 2536 in Fig. 15. These diagrams were made at the locations of the two slice lines cutting across the southern part of the system in Fig. 13. The abscissa in Fig. 15 is along these slice lines with the northeastern side on the left in each P-V diagram. The upper left panel and the two lower panels of Fig. 15 display $\mathrm{P}-\mathrm{V}$ diagrams at the location of the slice line through the optical nucleus of NGC 2536 along the photometric major axis of NGC 2536 at $\mathrm{PA}=38^{\circ}$; the upper right panel is a $\mathrm{P}-\mathrm{V}$ diagram at the location of the parallel slice line cutting across the bridge north of NGC 2536 and will be used for comparison. The upper left panel displays the combined $\mathrm{H}$ I emission from the bridge and NGC 2536. The plus sign marks the optical position and systemic velocity of the nucleus of NGC 2536. Three apparent velocity features are labeled in this panel. From comparison with the P-V dia- gram made farther north along the bridge (and shown in the upper right panel), the quasi-horizontal feature 1 is gas that belongs to NGC 2535, mainly bridge gas.

To understand features 2 and 3, we first extracted feature 3 from the cube and made moment maps. The upper left panel of Fig. 16 displays a high resolution $N(\mathrm{H} \mathrm{I})$ image of feature 3 in greyscale overlaid by contours of the total $\mathrm{H} \mathrm{I}$ column density remaining after feature 3 was removed. The plus sign marks the location of the optical nucleus of NGC 2536. Except for a fainter extension SW of the nucleus, the $\mathrm{H} \mathrm{I}$ emission from feature 3 corresponds in position to the $B$-band image of the northeastern half of NGC 2536 and in velocity to the $\mathrm{H} \alpha$ velocities (from Amram et al. 1989) on the northeastern side. In the NE half of NGC 2536, the $\mathrm{H} \alpha$ velocity field has $v=4035-4095 \mathrm{~km} \mathrm{~s}^{-1}$, and the $\mathrm{H}$ I cube of feature 3 has emission over the range $4009-4089 \mathrm{~km} \mathrm{~s}^{-1}$. The low spatial resolution of the $\mathrm{H}$ I data precludes a detailed comparison between the $\mathrm{H} \alpha$ and $\mathrm{H}$ I velocity fields here; the $\mathrm{H}$ I velocity field of feature 3 has $v=4039-4066 \mathrm{~km} \mathrm{~s}^{-1}$ on this side. We are fairly confident that most of feature 3 is $\mathrm{H} \mathrm{I}$ in the northeastern half of the disk of NGC 2536. The H I mass of feature 3 is $3 \times 10^{8} \mathscr{M}_{\odot}$, and the location of the maximum $N(\mathrm{H} \mathrm{I})$ in feature 3 coincides approximately with the very bright optical emission-line knot $5 " \mathrm{NE}$ of the 

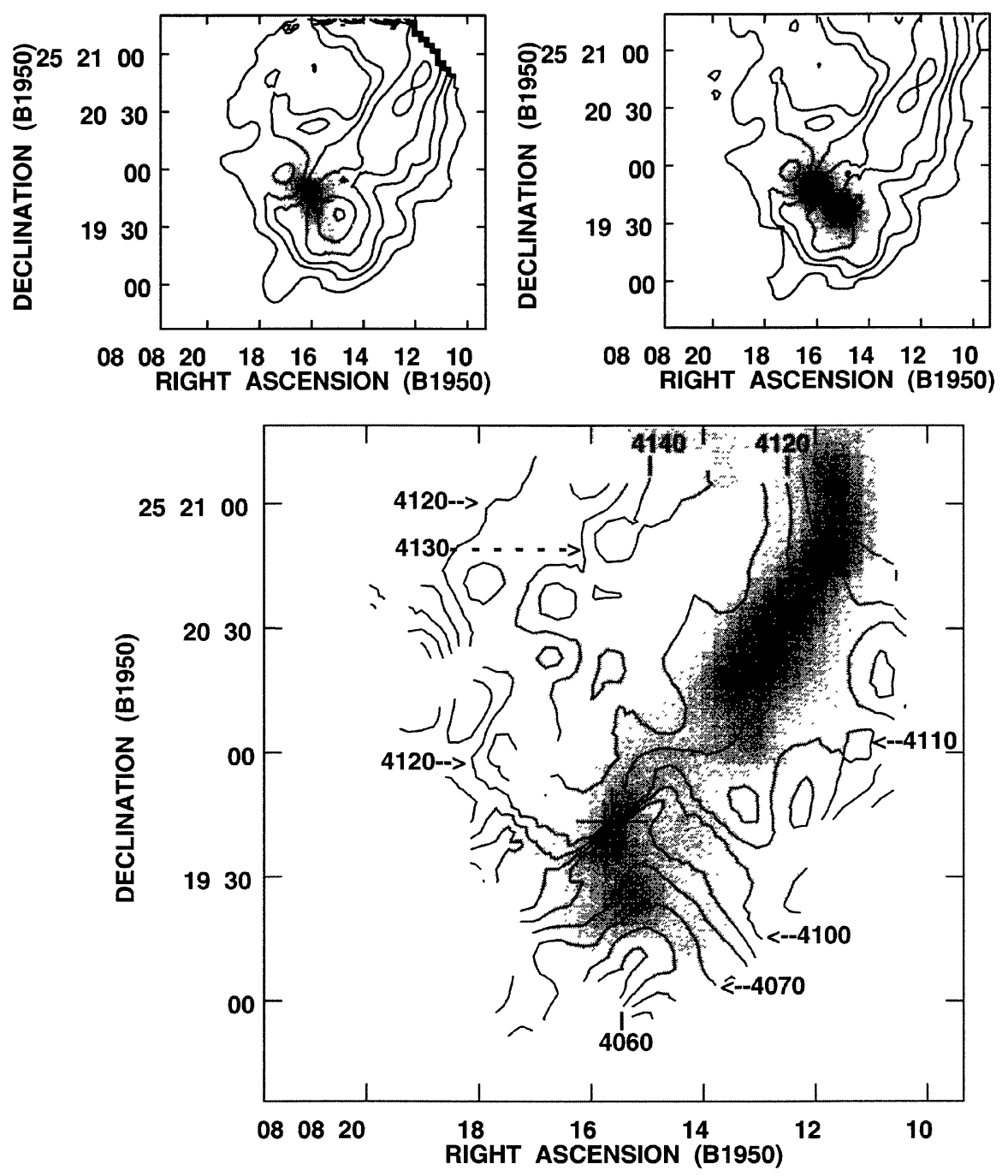

FIG. 16. $\mathrm{H} \mathrm{I}$ in the direction of NGC 2536 and the tidal bridge of NGC 2535. The plus sign marks the location of the optical nucleus of NGC 2536. Upper Panels: $N(\mathrm{H} \mathrm{I})$ associated with NGC 2536 in greyscale and the residual $N(\mathrm{H} \mathrm{I})$ from the tidal bridge as contours. The contour levels are $4.2,7.5,10,14,18$, and $23 \times 10^{20}$ atoms $\mathrm{cm}^{-2}$. In the upper left panel, only the $\mathrm{HI}$ emission from feature 3 (mainly the northeastern half of NGC 2536) is attributed to NGC 2536, whereas the upper right panel uses a symmetrized version of NGC 2536 (see text). Lower Panel: Isovelocity contours of the bridge gas overlaid on the H I column density of the bridge gas in greyscale after subtraction of the symmetrized version of NGC 2536 . The contour interval is $10 \mathrm{~km} \mathrm{~s}^{-1}$.

nucleus. The bright $\mathrm{H} \alpha$ emission from NGC 2536 is strongly one-sided and dominated by the NE knot.

As a guide for interpreting the rest of the $\mathrm{H} \mathrm{I}$ emission in the upper left panel of Fig. 15, we refer to the $\mathrm{H} \alpha$ velocity field of NGC 2536 in Amram et al. (1989). The $\mathrm{H} \alpha$ velocity field is that of a rotating disk with some distortions; it has $v_{\text {sys }}=4085 \pm 10 \mathrm{~km} \mathrm{~s}^{-1}$, kinematic major axis at PA $=16-23^{\circ}$ (differs between north and south), and its receding side is on the south. The $\mathrm{HI}$ in the part of feature 3 that appears southwest of the NGC 2536 nucleus in the $N(\mathrm{H} \mathrm{I})$ image in the upper left panel of Fig. 16 has a velocity of $\sim 4040 \mathrm{~km} \mathrm{~s}^{-1}$. This is probably not $\mathrm{H} \mathrm{I}$ in the disk of NGC 2536, since the $\mathrm{H} \alpha$ velocities (from Amram et al.) in the southwestern disk of NGC 2536 are $4105-4145 \mathrm{~km} \mathrm{~s}^{-1}$. The lower left panel of Fig. 15 displays the residual emission after feature 3 was removed from the cube. The residual emission in the upper half of feature 2 is low velocity gas $\left(v=4040-4080 \mathrm{~km} \mathrm{~s}^{-1}\right.$ ) some $25^{\prime \prime} \mathrm{SW}$ of the optical center of NGC 2536 and, thus, is also not gas in the main disk of NGC 2536. In Klarić's (1993) model, NGC 2536 is on the near side (relative to us) of much of the bridge gas here. Therefore this low velocity H I southwest of the NGC 2536 nucleus could represent gas near the end of the bridge streaming towards NGC 2536 from the far side and in a retrograde sense relative to the rotation of NGC 2535. This would be consistent with Klarić's model, which has NGC 2536 accrete gas from NGC 2535. By "'bridge gas," we mean gas not in the main disk of NGC 2536; it may include gas falling into or being ejected from NGC 2536.

The emission in the bottom half of feature 2 in Fig. 15 has $v=4110-4155 \mathrm{~km} \mathrm{~s}^{-1}$ at spatial positions corresponding to the northeastern disk of NGC 2536. This is high compared to the $\mathrm{H} \alpha$ velocities in the northeastern disk of NGC 2536 but 
similar to the velocities farther north in the bridge. This is probably bridge gas. Farther west in the bottom half of feature 2 , the bridge gas blends with gas in the southwestern disk of NGC 2536. The combination of the two may account for the strong peak at $4116 \mathrm{~km} \mathrm{~s}^{-1}$ in feature 2 .

The southwestern disk of NGC 2536 appears embedded in the bridge gas (i.e., same position and same velocity) and is not extractable without an additional assumption. Suppose we cut the emission from feature 3 along the photometric minor axis of NGC 2536 at $\mathrm{PA}=128^{\circ}$ and assume inversion symmetry for the $\mathrm{H}$ I in NGC 2536, relative to the optical nucleus. The upper right panel in Fig. 16 displays the resulting $N(\mathrm{H} \mathrm{I})$ image of NGC 2536 in greyscale and the residual $N(\mathrm{H} \mathrm{I})$ emission from the bridge as contours, both for the low resolution images. The $\mathrm{HI}$ mass of the symmetrized NGC 2536 is $4 \times 10^{8} \mathscr{O}_{\odot}$. In these low resolution images, the peak H I column density in the disk of NGC 2536 is $1.0 \times 10^{21}$ atoms $\mathrm{cm}^{-2}$, and the peak $\mathrm{H}$ I column density in the bridge gas in the direction of NGC 2536 is 1.8 $\times 10^{21}$ atoms $\mathrm{cm}^{-2}$, so, under the assumed symmetry, the bridge gas is the dominant component. Here "bridge gas" means the residual after subtracting the symmetrized disk of NGC 2536.

Assuming that the velocities as well as the distribution of $\mathrm{HI}$ in NGC 2536 have inversion symmetry, we subtracted the symmetrized NGC 2536 emission from the original $\mathrm{H}$ I cube to obtain the cube of emission from the bridge gas and its velocity field. Taking the central velocity of NGC 2536 as $4085 \mathrm{~km} \mathrm{~s}^{-1}$ produced an unlikely hole in the $\mathrm{P}-\mathrm{V}$ diagrams of the bridge gas, so we took the central velocity of NGC 2536 as $4097 \mathrm{~km} \mathrm{~s}^{-1}$; this resulted in more plausible $\mathrm{P}-\mathrm{V}$ diagrams for the bridge gas. Recall that the optical $v_{\text {sys }}$ is $4085 \pm 10 \mathrm{~km} \mathrm{~s}^{-1}$. The lower right panel of Fig. 15 displays the resulting $\mathrm{P}-\mathrm{V}$ diagram of the bridge gas for the same field as in the upper left panel (which shows the combined emission from the bridge plus NGC 2536). The resulting velocity field of the bridge gas is plotted in the lower panel of Fig. 16. The steep velocity gradient in the bridge gas at the location of NGC 2536 is probably an artifact of the symmetrization process and suggests that the assumption of symmetry in the velocity field of NGC 2536 and/or the assumption of symmetry in the distribution of $\mathrm{H} \mathrm{I}$ in NGC 2536 are incorrect.

In fact, computer simulations for this type of encounter (Curtis Struck, in progress) do not predict inversion symmetry. The accretion of gas onto NGC 2536 may have been one-sided, and the accreted gas may be still in threedimensional orbits. In Klarić's (1993) model, shocks in the bridge gas occur south or southwest to west of NGC 2536, there is a stream of material from NGC 2535 swinging from below the disk plane of NGC 2536 and a large fan-shaped feature behind and east of NGC 2536, and all this material is quickly dumped onto NGC 2536. In Fig. 16, we see that the main $N(\mathrm{H} \mathrm{I})$ clump in the bridge gas is centered $12^{\prime \prime} \mathrm{SW}$ of the nucleus of NGC 2536 and has a velocity of $4075 \mathrm{~km} \mathrm{~s}^{-1}$; this low velocity gas may correspond to some of the shocked gas in Klarić's model.

In summary, we have successfully separated the $\mathrm{H}$ I emission of the northeastern disk of NGC 2536 from the bridge
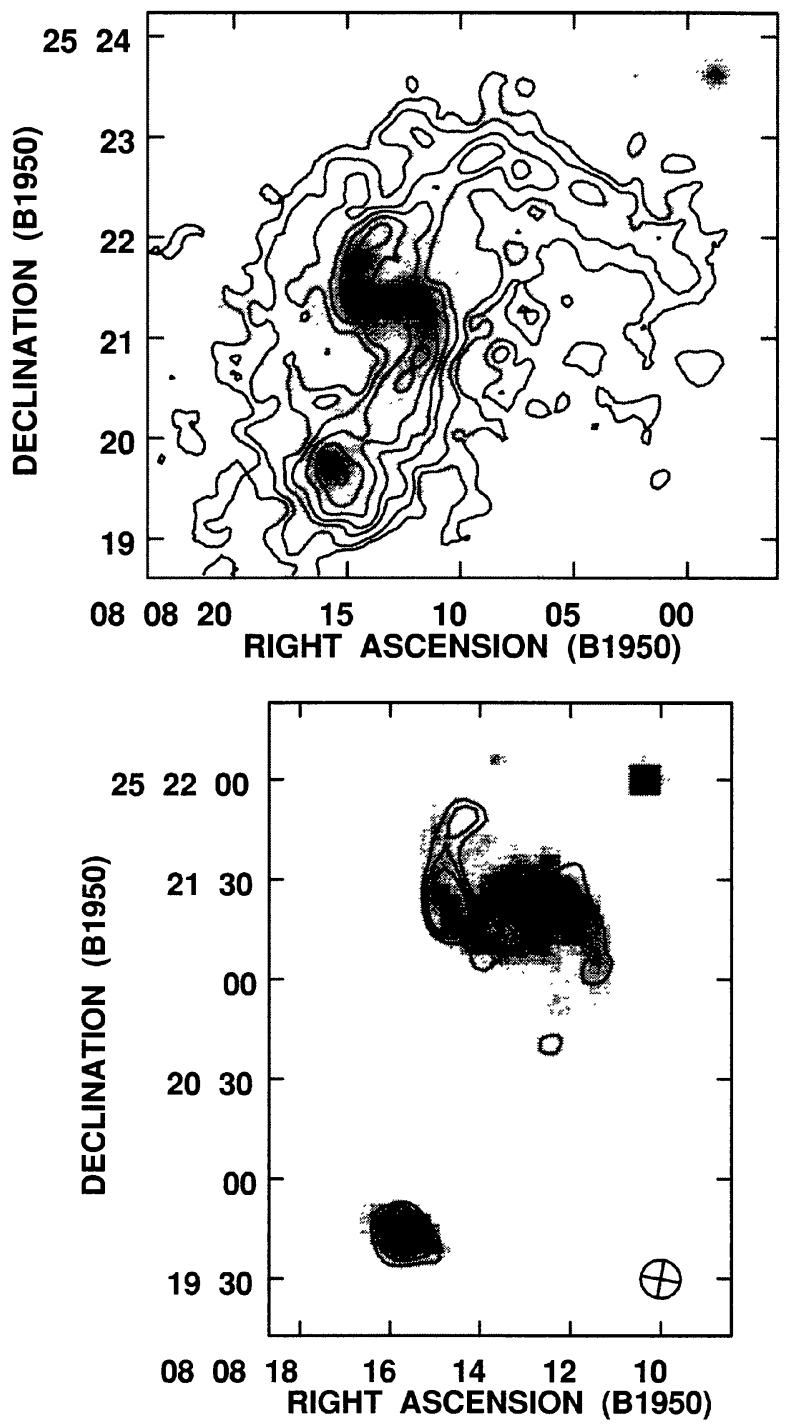

FIG. 17. Top: The low resolution $\lambda=20 \mathrm{~cm}$ radio continuum image of both galaxies in greyscale with $N(\mathrm{H} \mathrm{I})$ contours from Fig. 7. Bottom: The high resolution $\lambda=20 \mathrm{~cm}$ radio continuum image as contours overlaid on the $B$-band image in greyscale. The beam symbol represents the radio continuum beam size $\left(12.0^{\prime \prime} \times 11.5^{\prime \prime}\right)$. The rms noise is $0.19 \mathrm{mJy}^{\mathrm{beam}}{ }^{-1}$, or $0.86 \mathrm{~K}$, and the contours levels are at $3,4,5$, and $10 \times$ the rms noise. Notice the bar-like feature in the radio continuum emission from NGC 2535.

gas and have also found a component of the bridge gas southwest of the NGC 2536 nucleus that may represent gas streaming towards NGC 2536. The southwestern disk of NGC 2536 has the same projected position and the same velocity as some of the bridge gas there. Since the northeastern half of NGC 2536 has an H I mass of $2 \times 10^{8} \mathscr{M}_{\odot}$, the total H I mass of NGC 2536 is at least $2 \times 10^{8} \mathscr{M}_{\odot}$ and may be $\sim 4 \times 10^{8} \mathscr{O}_{\odot}$ if the distribution of $\mathrm{H} \mathrm{I}$ in NGC 2536 is roughly symmetric. Unless the $\mathrm{H}$ I mass of the southwestern half of NGC 2536 is appreciably greater than that of the northwestern half, the dominant H I column density in the direction of NGC 2536 is the bridge gas.

Recall from Sec. 4 that our nondetection of ${ }^{12} \mathrm{CO}$ emission from NGC 2536 gives an upper limit of $6 \times 10^{8} \mathscr{l l}_{\odot}$ for 
the $\mathrm{H}_{2}$ mass of NGC 2536. Then the mass of molecular gas in the starburst galaxy NGC 2536 is, at most, three times the $\mathrm{H}$ I mass.

\section{RADIO CONTINUUM RESULTS}

The total $\lambda 20 \mathrm{~cm}$ radio continuum flux density $S_{\nu}(20)$ of the system is $46 \mathrm{mJy}$, of which $6.2 \mathrm{mJy}$ is from the starburst galaxy NGC 2536. The top panel of Fig. 17 displays the low resolution $\lambda 20 \mathrm{~cm}$ continuum image (resolution $=15.1^{\prime \prime}$ $\times 14.6^{\prime \prime}$ FWHM) in greyscale with $N(\mathrm{H} \mathrm{I})$ contours overlaid, and the bottom panel displays the high resolution $\lambda 20 \mathrm{~cm}$ continuum image (resolution $=12.0^{\prime \prime} \times 11.5^{\prime \prime} \mathrm{FWHM}$ ) as contours overlaid on the $B$-band image in greyscale. In the radio continuum, NGC 2535 looks like a barred spiral with a barlike feature connecting the two apices of the eye-shaped oval at $\mathrm{PA}=100^{\circ}$. The most luminous blue and $\mathrm{H} \alpha$ knots occur at or near these two apices. The radio continuum 'bar' leads the weak stellar bar by $\approx 50^{\circ}$, a surprisingly large angle. The high resolution optical image in Arp (1969) gives no indication of a dust lane along the radio continuum bar. Instead, one sees a curved dust lane cutting across just north of the nucleus from the NE rim of the eye-shaped oval to the inside edge of the southern arm of NGC 2535, with position angles in the range $65^{\circ}$ to $75^{\circ}$. The dust-lane morphology is hard to explain if the radio continuum bar is simply a shock front in the gas on the leading side of a stellar bar.

Perhaps the weak stellar bar is the remnant of an older bar, whereas the radio continuum "bar"' in NGC 2535 may be shocked gas from a new bar in the process of formation. Or perhaps the radio continuum "bar" is a two-sided jet from the nucleus. Bernlöhr (1993) classifies the optical emission lines as $\mathrm{H}$ II region-type, not Seyfert or LINER. The nucleus of NGC 2535 has a $\lambda 20 \mathrm{~cm}$ continuum surface brightness of only $1.6 \mathrm{mJy}_{\text {beam }}^{-1}$ on the high resolution image and is somewhat fainter in radio continuum surface brightness than the eastern apex of the oval (where there are two bright knots in the $B$-band image). Hence, the jet interpretation of the radio continuum bar-like feature appears less likely.

The radio continuum emission in NGC 2535 comes from the radio continuum bar, the nucleus, the two apices of the oval, and the portions of the $\mathrm{H}$ I tidal arms relatively close to the eye-shaped oval. The rim of the eye-shaped oval in NGC 2535 is not clearly outlined in $\lambda 20 \mathrm{~cm}$ continuum and $\mathrm{H} \mathrm{I}$ emission as it is in IC 2163 (compare the NGC 2535 images here with the IC 2163 images in Condon 1983 and Paper I). This suggests that in NGC 2535 the rim is not presently a strong shock front, unlike the situation in IC 2163. On the tidal tail of NGC 2535 , the radio continuum emission, like the $\mathrm{HI}$ emission, lies between the main optical arm and the spur.

In the high resolution $\lambda 20 \mathrm{~cm}$ continuum image, the center of NGC 2536 has a flux density of $1.2 \mathrm{mJy}$ and a peak surface brightness of $1.7 \mathrm{mJy}_{\text {beam }}{ }^{-1}$, about the same as the nucleus of NGC 2535. If this is optically-thin free-free emission, it is equivalent to 19 times the most luminous $\mathrm{H} \mathrm{II}$ region (CCM 72) in M51 (van der Hulst et al. 1988). The radio continuum peak of NGC 2536 is at the same location
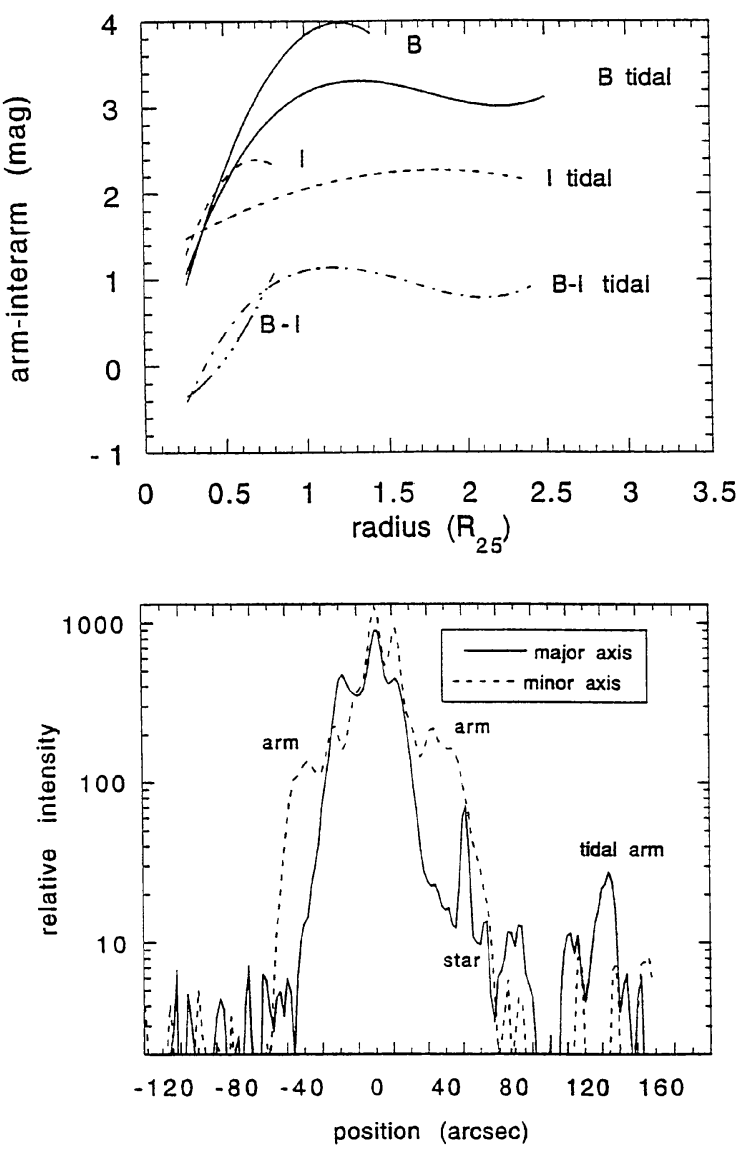

FIG. 18. Top: Arm/interarm contrast in $B$ band (solid curves) and $I$ band (dashed curves) as a function of radius (in units of $R_{25}$ ) in the face-on image of NGC 2535. The curves for the tidal tail are labelled "tidal"; the other curves represent the tidal bridge. Bottom: Radial profile of the face-on $B$ image of NGC 2535 for cuts along the kinematic major axis at $\mathrm{PA}=153^{\circ}$ (solid curves, with positive positions on the NW side of the galaxy) and the kinematic minor axis at $\mathrm{PA}=63^{\circ}$ (dashed curves, with positive positions on the NE side). On the cut along the kinematic minor axis, the tidal arms are labelled "arm," with the tidal bridge at the negative positions and the tidal tail at the positive positions. The feature labelled "tidal arm" on the cut along the kinematic major axis is the tidal tail near its sharp bend.

as the optical peak in the $B$ and $I$-band images.

\section{OPTICAL SURFACE PHOTOMETRY OF NGC 2535}

The arm-to-interarm contrasts as a function of radius in the deprojected, sky-subtracted $B$-band and $I$-band images are displayed in the top panel of Fig. 18. We used the projection parameters from Table 4 and assumed that the disk is flat. The arm-interarm contrast is less extreme than Paper I found in IC 2163, but still large compared to normal spiral arms, which have a typical arm contrast of 1-2 mag (see Elmegreen \& Elmegreen 1984). The tidal tail can be measured out to $R=2.7 R_{25}=200^{\prime \prime}$ in $B$ band and in H I. In $B$ band, the interarm near the tidal tail is measured with an accuracy of $\pm 0.1 \mathrm{mag}$ out to $R=2.0 R_{25}$ but blends into the sky background by $R=2.5 R_{25}$. The maximum value for the arm contrast is $4.2 \mathrm{mag}$ and occurs at $R=1.2 R_{25}$. Beyond this, the arm contrast on the long tidal tail averages 3.2 mag 


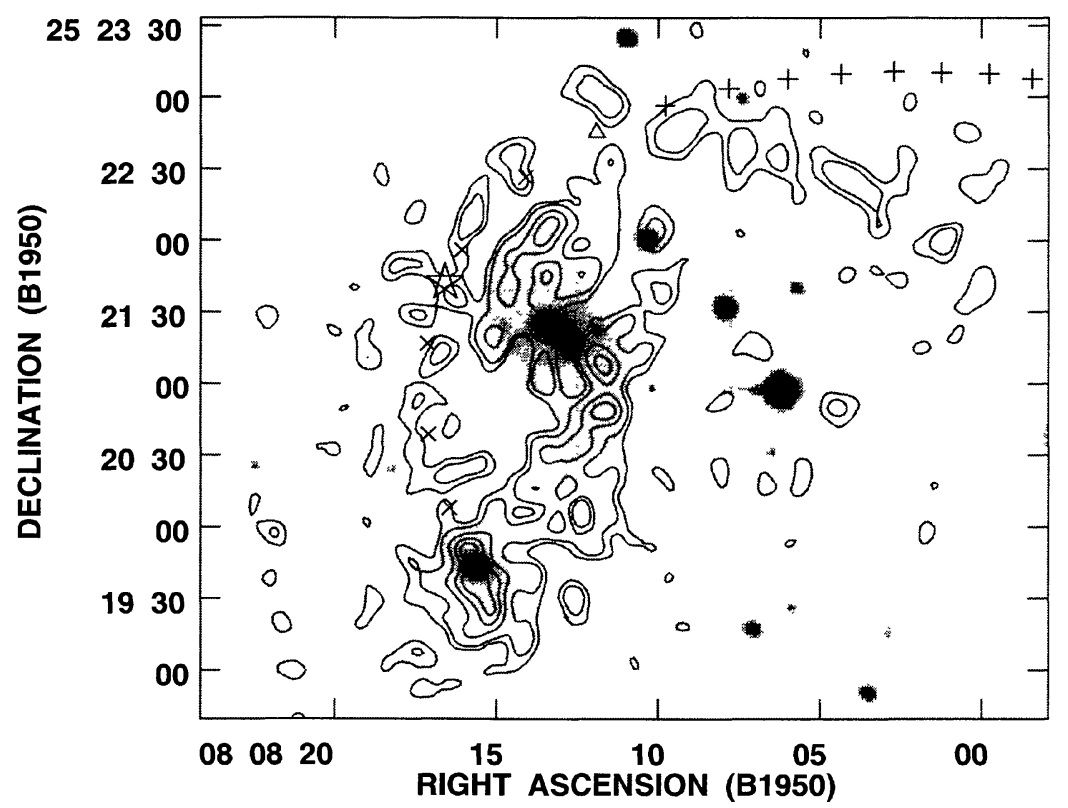

FIG. 19. The sky-plane projection of Klarić's (1993) orbit for NGC 2536 with +'s for the portion of the orbit above the initial disk plane of NGC 2535, $x$ 's for the portion of the orbit below the disk plane, a triangle at the disk crossing from near to far (relative to us), and a five-pointed star at perigalacticon. The orbit is overlaid on a contour plot of the high resolution $N(\mathrm{H} \mathrm{I})$ image (with the same contour levels as in Fig. 5) and the $I$-band image in greyscale.

and is fairly constant. Throughout the main disk (i.e., $R$ $\leqslant R_{25}$ ) the arm-interarm contrast generally increases with radius. This behavior suggests that the arms here have a tidal origin, since in density-wave galaxies, the contrast tends to level off or decrease after about $0.5 R_{25}$ [see Fig. 6 in Elmegreen et al. (1996) for the arm contrast in barred galaxies]. Although galaxy encounter simulations, such as those in Elmegreen et al. (1991) and Paper II, predict a large stellar arm-interarm contrast for tidal arms at a certain stage of the interaction, we are unaware of other studies (aside from $\mathrm{Pa}$ per I) in which the stellar arm contrast of tidal arms has been measured. Hibbard (1995) comments that the tails in NGC $4676 \mathrm{a} / \mathrm{b}$ have high surface brightness in the $R$-band image.

The bottom panel of Fig. 18 displays radial profiles of the deprojected $B$-band image for cuts along the kinematic major and kinematic minor axes. The rim of the eye-shaped oval is prominent at $R=15^{\prime \prime}-25^{\prime \prime}$. Beyond the rim of the eyeshaped oval on the kinematic major axis and after crossing the tidal arms at $R=30^{\prime \prime}-50^{\prime \prime}$ on the kinematic minor axis, the surface brightness declines very steeply. This very steep decline is unusual and indicates that most of the stars have collected in the eye-shaped oval and the tidal arms. Since the interarm disk beyond $R=60^{\prime \prime}$ is detected, not all of the stars have been cleared out of the interarm by the interaction. The cut along the kinematic minor on the NE side crosses the elliptical arc at 52" in the sky-plane, which corresponds to a disk position in Fig. 18 of $+55^{\prime \prime}$. As no local peak in the $B$-band radial profile is visible at this location, the elliptical arc appears to be a purely gaseous feature. The prominent feature labelled "tidal arm" on the cut along the kinematic major axis is the tidal tail near its sharp bend.

\section{WHAT IS THE ORIGIN OF THE H I ELLIPTICAL ARC?}

The elliptical arc is a gaseous structure; it is not apparent in the $B$-or $I$-band images. On the eastern side of NGC 2535 , the elliptical arc is a clear feature in the $N(\mathrm{H} \mathrm{I})$ image and has projected peculiar velocities of $10-20 \mathrm{~km} \mathrm{~s}^{-1}$ receding from us relative to the neighboring gas in the disk of NGC 2535. It is a long structure, stretching from the north around counterclockwise to the southeast, but stops some distance from NGC 2536. The apparent extension of the elliptical arc on the western side of NGC 2535 is hard to trace in the $N(\mathrm{H} \mathrm{I})$ image but is evident as a structure in the velocity field from the west around to the northwest with projected peculiar velocities of $10-20 \mathrm{~km} \mathrm{~s}^{-1}$ approaching us.

Elmegreen et al. (1992) showed that pre-existing outer resonance rings are destroyed by nearby encounters. On the other hand, if the elliptical arc were an expanding ring in the disk-plane of NGC 2535, the velocity perturbations would be opposite to those observed. On the NE kinematic minor axis (which is the near side of NGC 2535), an expanding ring would produce peculiar velocities approaching us relative to the undisturbed disk whereas the observed peculiar velocities of the elliptical arc are receding from us here. Therefore, the elliptical arc is not an expanding wave-like shell in the disk of NGC 2535.

A more promising interpretation is suggested by Klarić's (1993) orbit for NGC 2536. The symbols in Fig. 19 represent this orbit after projection into the sky-plane, with + 's for the portion of the orbit above the initial disk-plane of NGC 2535, $x$ 's for the portion below the initial disk-plane, a triangle at the disk-crossing from the near side to the far side, and a five-pointed star at perigalacticon; the symbols are 
TABLE 6. Comparison of the ocular galaxies NGC 2535 and IC 2163.

\begin{tabular}{|c|c|c|}
\hline Property & NGC 2535 & IC 2163 \\
\hline 1. Central eye-shaped oval with intrinsically oval shape & Yes & Yes \\
\hline 2. Long tidal arms in optical and $\mathrm{HI}$ & Yes & Yes \\
\hline 3. Nearly constant velocity along much of the tidal tail & Yes & Yes \\
\hline 4. Streaming motions around the eye-shaped oval & Yes & Yes \\
\hline \multicolumn{3}{|l|}{ 5. Stellar arm contrast of tidal arms is large } \\
\hline compared to normal spiral arms & Yes & Yes \\
\hline 6. Widespread, high velocity dispersion in the HI gas & Yes & Yes \\
\hline 7. Massive $\left(10^{8} \mathrm{M}_{\odot}\right)$ HI clouds in the disk but & & \\
\hline not associated with major stellar clumps & Yes & Yes \\
\hline \multicolumn{3}{|l|}{ 8. $M_{\text {vir }}$ of the massive clouds is an order of magnitude } \\
\hline greater than $M(H I)$ & Yes & Yes \\
\hline 9. Small, weak stellar bar & Yes & Yes \\
\hline \multicolumn{3}{|l|}{ 10. Double-parallel arm structure on tidal tail with } \\
\hline$\sim 100 \mathrm{~km} \mathrm{~s}^{-1}$ velocity difference between the two components & No & Yes \\
\hline 11. Rim of eye-shaped oval outlined by HI and & & \\
\hline radio continuum emission & No & Yes \\
\hline \multicolumn{3}{|l|}{ 12. Radio continuum bar-like feature that leads stellar bar } \\
\hline by large angle & Yes & No \\
\hline 13. Ocular galaxy more massive than companion & Yes & No \\
\hline 14. Starburst companion & Yes & No \\
\hline 15. Extended HI and stellar envelope & Yes & No \\
\hline 16. $z$-motions in outer disk of ocular & Yes & No \\
\hline 17. Outer elliptical HI arc & Yes & No \\
\hline
\end{tabular}

separated by time steps of $28 \mathrm{Myr}$ (except for perigalacticon) and are overlaid on a contour plot of the high resolution $N(\mathrm{H} \mathrm{I})$ image with the $I$-band image in greyscale. Klaric determined this orbit to reproduce the observed stellar tidal features. This orbit was selected before the $\mathrm{H}$ I observations were made and was not adjusted to fit the $\mathrm{HI}$ data. The elliptical arc on the eastern side of NGC 2535, from the north around counterclockwise to the southeast, coincides with the sky-projection of Klarić's orbit. This suggests that the elliptical arc on this side is a wake in the gas produced by the passage of the companion within or close to the extended $\mathrm{H}$ I envelope.

Donner \& Thomasson (1993) evaluated the following mechanisms for getting a purely gaseous structure along the orbit of a companion, as possible explanations for the North Tidal Bridge in the M81 triplet: (a) ram-pressure stripping in which gas clouds in the companion collide with gas clouds in the primary, (b) tidal stripping of gas from the companion, and (c) a gravitational wake in which gas clouds in the extended gas disk of the primary are accelerated towards the companion, collide inelastically, and collect behind the companion. In the case of M81, they found that ram-pressure stripping does not produce a tail of material staying near the orbit of the companion, tidal stripping can produce such a tail but not a very long one, whereas the gravitational wake in their M81 model lies nearly along the orbit of the companion for about $90^{\circ}$. A gravitational wake can be created even when the orbit of the companion is slightly inclined with respect to the disk of the primary. It results from the collision of gas clouds from inside and outside of the companion's orbit and thus requires that the disk of the primary extend beyond the orbit of the companion.

Because NGC 2535 has a sufficiently extended H I envelope and NGC 2536 appears to have been a spiral galaxy before the interaction, it seems likely that all three of the above mechanisms are in operation in NGC 2535/36. The H I scale height of the extended envelope in NGC 2535 should be large because of the high $\mathrm{H}$ I velocity dispersion and the usual flaring of the disk when the total mass surface decreases [see Eq. (5)]. We shall assume that the main process creating the long elliptical arc on the eastern side of NGC 2535 is the gravitational wake. Since in ocular models the orbit of the companion is nearly in-plane and prograde, the gas in the envelope is already orbiting in the same sense as the companion and merely needs to be compressed by inelastic collisions to form a gravitational wake. On the eastern side of NGC 2535, the orbit of NGC 2536 is below the disk-plane of NGC 2535 (i.e., on the far side relative to us). This gives the correct sense of velocity perturbation to the wake: the observed peculiar velocities of the elliptical arc are receding here. Since the projected peculiar velocities are only $10-20 \mathrm{~km} \mathrm{~s}^{-1}$, and the inclination of the present disk of NGC 2535 is $23^{\circ} \pm 4^{\circ}$ with respect to the sky-plane, the tilt of the wake relative to the present disk of NGC 2535 and the sky-plane should be fairly small. If gas clouds in the wake were to follow the same orbit as NGC 2536, then the angular velocity of these clouds should be less than the angular velocity of NGC 2536 (e.g., in a point-mass model, by a factor of $\left[\mathscr{C}_{1} /\left(\mathscr{C}_{1}+\mathscr{C}_{2}\right)\right]^{1 / 2}$, where $\mathscr{M}_{1}$ is the mass of NGC 2535 and $\mathscr{C l}_{2}$ is the mass of NGC 2536). The clouds in the wake are probably not following exactly the same orbit as NGC 2536, just one that lies close to it in projection. Since it takes time for the accelerated clouds to collide and produce a gravitational wake, we expect such a wake to stop some distance behind the companion, as, indeed, is observed for the elliptical arc. The string of faint $\mathrm{H} \alpha$ emission from the north counterclockwise to the NE along the elliptical arc could be from some new star formation in the compressed gas (no local peak is seen in the $B$-band radial profile because the integrated $B$ light is from older stars than the $\mathrm{H} \alpha$ emission) or from shock-ionized gas in the wake.

Klarić's (1993) simulations did not predict a wake. To 
produce a gravitational wake, a model must start with an extended gas disk and include sufficient energy dissipation for the wake to hold together. Klarićs simulations did not include an extended gas disk or allow for inelastic collisions in the gas. An extended gas disk and inelastic collisions will be added to the models in a paper to be published later.

A gravitational wake is not a plausible explanation for the apparent weak extension of the elliptical arc on the western side of NGC 2535. This feature, which is visible mainly in the velocity field, must have formed by some other mechanism.

\section{DISCUSSION AND CONCLUSIONS}

In Table 6, we compare the properties of the eye-shaped (ocular) galaxy NGC 2535 with those of the ocular galaxy IC 2163 studied in Paper I. Properties 1-5 (the morphology, the streaming motions around the eye-shaped oval, the constant velocities along the tidal arms, and the large values for the stellar arm contrast) occur in both galaxies and are general ocular characteristics predicted by computer simulations of prograde, in-plane galaxy encounters.

Properties 6 and 7 (the enhanced turbulence in the gas and the unusually massive $\mathrm{H}$ I clouds not associated with major clumps in the older stellar population) are found in the nonocular interacting galaxies NGC 2207 (Paper I) and NGC 5774 (Irwin 1994) as well as in the ocular galaxies NGC 2535 and IC 2163, and thus appear to be general features of a particular early phase of post-encounter evolution, independent of the orientation and sense of the galaxy orbit. The widespread high velocity dispersion in the $\mathrm{H}$ I gas could result from collisions between tidally-accelerated clouds. It is not seen in NGC 7714/15 (Smith 1997b), in the western tail of NGC 2782 (Smith 1997a), or in the tidal tails of the five galaxy-merger pairs studied by Hibbard (1995). These systems are in a later stage of interaction, e.g., NGC 7715 is classified as post-starburst and the merger pairs are more evolved interactions, so perhaps the turbulence generated by the tidal agitation of the gas has already dissipated.

Almost none of the massive $\mathrm{H}$ I clouds in NGC 2535, IC 2163, NGC 2207, NGC 5774 (see Irwin 1994), or on the western tail of NGC 2782 (see Smith 1994) contains an unusually bright clump of star formation. Perhaps these clouds are too recently formed to have produced many new stars. Alternatively, because of the high velocity dispersion, the scale height of the gas is large and, hence, the gas volume density is not particularly high; this would increase the collapse time and delay star formation.

In NGC 2535, the five massive clouds have virial masses $7-11$ times greater than the $\mathrm{H}$ I masses. From the ${ }^{12} \mathrm{CO}$ observations, we conclude that in, at least, four of the five clouds, the molecular mass is insufficient to explain the difference. Thus either (a) the stellar contribution to $\mathscr{M b}_{\text {vir }}$ is important because the H I scale height is large, or (b) the clouds are not virialized, or (c) there is a significant contribution to the $\mathrm{HI}$ velocity dispersion from velocities other than turbulence. In NGC 2535, the stellar surface density suffices to account for the difference between $\mathscr{C}_{\text {vir }}$ and $\mathscr{M}$ (H I); we conclude that these are simply thick clouds as a result of the high velocity dispersion in the gas. Using the data from Paper I, we find that ten of the eleven massive $\mathrm{H} \mathrm{I}$ clouds in IC 2163/NGC 2207 have virial masses 6-23 times greater than the $\mathrm{H}$ I masses. The exception, Cloud 12 , near the outer end of the bright part of the $\mathrm{H}$ I tidal tail of IC 2163, has $\mathscr{M b}_{\text {vir }}=2.4 \mathscr{L}$ ( $\mathrm{H} \mathrm{I}$ ), the lowest velocity dispersion of the 11 clouds, and thus, probably, the smallest H I scale height. Our mapping of the ${ }^{12} \mathrm{CO}$ emission from IC 2163/ NGC 2207 is in progress. A number of the massive clouds in this interacting pair are in the main optical disk, and hence the stellar contribution to $\mathscr{L}_{\text {vir }}$ could be important. On the other hand, Cloud N1 in NGC 2207 lies in the outer part of the disk where there are large $z$ motions creating the warp and where the stellar surface density is low. Whereas explanation (a) for the difference between $\mathscr{M}_{\text {vir }}$ and $\mathscr{C}(\mathrm{H}$ I) is suitable for many of the other clouds, it does not apply to Cloud N1.

The double-parallel arm structure (tidal tail plus streaming arm) seen on the anti-companion side in IC 2163 but not in NGC 2535 is a very short-lived feature in the models. In Klarić's (1993) encounter model for NGC 2535/36, the two components of the tidal tail have already merged. Thus, the differences between NGC 2535 and IC 2163 in properties $10-12$ probably mean that we are viewing NGC 2535 at a slightly later epoch of post-encounter evolution than IC 2163. In the models, perigalacticon occurred $40 \mathrm{Myr}$ ago in the IC 2163/NGC 2207 pair (Paper II) and $100 \mathrm{Myr}$ ago in Klarić's model for NGC 2535/36. Paper I finds a compressed ridge of rapidly streaming gas along the rim of the eyeshaped oval in IC 2163, but a similar compression is not evident in our radio continuum and $\mathrm{H}$ I observations of NGC 2535. Instead, NGC 2535 has a bar-like radio continuum feature that connects the two apices of the eye-shaped oval and leads the stellar bar by $\sim 50^{\circ}$. There is no indication of a dust lane along the radio continuum bar. The radio continuum bar may be a two-sided jet from the nucleus, or it may be a new bar in the process of formation. In the latter case, this would be an example of bar formation following a strong encounter, as predicted by Noguchi (1987). In the Elmegreen et al. (1991) models, the double-parallel arm structure disappears before a new bar is formed.

As more time has elapsed since perigalacticon in the NGC $2535 / 36$ system than in the IC 2163/NGC 2207 system, this has given sufficient time for a starburst (Property 14) to develop in NGC 2536. In Klarić's encounter simulation, NGC 2536 accretes gas from NGC 2535, and this may have fueled the starburst. In the H I P-V diagrams of NGC 2536 and the tidal bridge of NGC 2535, we identify a feature that may represent bridge gas streaming towards NGC 2536, as in Klarić's model. Bernlöhr (1993) presents observational evidence that interaction-driven starbursts develop first in the less massive member of the galaxy pair. From the nearinfrared luminosities, we deduce an ocular/companion mass ratio of 2.8 for NGC 2535 and 0.6 for IC 2163. Using images from the digitized Ohio State University Galaxy Survey (Berlind et al. 1997), we find that the luminosity of IC 2163 in $H$ and $K$ bands is $60 \%$ of the luminosity of NGC 2207 (in the nonoverlapping halves). The model in Paper $\Pi$ for the 
encounter between IC 2163 and NGC 2207 adopted a 1:1 mass ratio for that system.

The substantial, extended H I envelope of NGC 2535 (Property 15) is important for supplying gas to the companion. IC 2163 may have started with a less extended envelope than NGC 2535. At present, NGC 2535 is surrounded by an extended $\mathrm{HI}$ and stellar envelope whereas the entire outer disk of IC 2163 seems to have been swept up into the tidal arms or to have fallen into the oval (as predicted by the simulation in Paper II). Perhaps this latter difference occurs because NGC 2535 is being perturbed by a companion of lower mass.

The evidence for $z$ motions in the outer part of NGC 2535 but not in IC 2163 (Property 16) indicates that the tilt of the orbit of NGC 2536 relative to the plane of NGC 2535 is nonzero, whereas the orbit of NGC 2207 is probably in the plane of IC 2163.

The long, outer, elliptical $\mathrm{HI}$ arc (Property 17) on the eastern side of NGC 2535 is a prominent gas feature not seen in IC 2163. The coincidence between the sky-projection of Klarić's (1993) orbit for NGC 2536 and the elliptical arc gives confirmation to Klarić's model. Klarić's orbit was cho- sen to reproduce the stellar tidal features and not subsequently adjusted to match the $\mathrm{H}$ I structures. The elliptical arc is most likely a gravitational wake produced by the passage of NGC 2536 within or close to the extended gaseous envelope of NGC 2535. The wake did not appear in Klarić's simulation of NGC 2535/36 because he did not include an extended gas disk or allow for inelastic collisions in the gas. IC 2163 does not have an outer elliptical H I arc; IC 2163 may not have possessed a sufficiently extended $\mathrm{H}$ I envelope. Donner \& Thomasson (1993) have modeled the North Tidal Bridge in the M81 triplet as a gravitational wake. The gravi tational wake in the M81 triplet and in NGC 2535/36 results from the smaller galaxy (NGC 3077 and NGC 2536, respectively) moving through the substantial, extended gas disk of the larger galaxy.

This research was partially supported by a Fullam-Dudley award to M.K. and by a grant to M.K. from NASA administered by the American Astronomical Society. Part of this work was done while M.K. visited the Astronomy Department, University of California at Berkeley, and she thanks the Astronomy Department at Berkeley for their hospitality.

\section{REFERENCES}

Amram, P., Marcelin, M., Boulesteix, J., \& Le Coarer, E. 1989, A\&AS, 81, 59

Arp, H. 1969, A\&A, 3, 418

Baars, J. W. M., Genzel, R., Pauliny-Toth, I. I. K., \& Witzel, A. 1997, A\&A, 61, 99

Barnes, J. E., \& Hernquist, L. 1992, Nature, 369, 715

Berlind, A. A., Quillen, A. C., Pogge, R. W., \& Sellgren, K. 1997, AJ, 114, 107

Bernlöhr, K. 1993, A\&A, 268, 25

Binney, J., \& Tremaine, S. 1987, Galactic Dynamics (Princeton University Press, Princeton), p. 67

Bothun, G. D., \& Schommer, R. A. 1982, AJ, 87, 1368

Chandrasekhar, S. 1969, Ellipsoidal Figures of Equilibrium (Yale University Press, New Haven)

Condon, J. J. 1983, ApJS, 53, 459

de Vaucouleurs, G., de Vaucouleurs, A, Corwin, Jr., H. G., Buta, R. J., Paturel, G., \& Fouqué, P. 1991, Third Reference Catalogue of Bright Galaxies (Springer, New York)

Dickey, J. H., Hanson, M. M., \& Helou, G. 1990, ApJ, 352, 522

Donner, K. H., \& Thomasson, M. 1993, A\&A, 279, 28

Elmegreen, B. G. 1989, ApJ, 338, 178

Elmegreen, B. G., Kaufman, M., \& Thomasson, M. 1993, ApJ, 412, 90

Elmegreen, B. G., Sundin, M., Kaufman, M., Brinks, E., \& Elmegreen, D. M. 1995b, ApJ, 453, 139 (Paper II)

Elmegreen, D. M., \& Elmegreen, B. G. 1984, ApJS, 54, 127

Elmegreen, D. M., Elmegreen, B. G., Combes, F., \& Bellin, A. D. 1992, A\&A, 257, 17

Elmegreen, D. M., Elmegreen, B. G., Chromey, F. R., Hasselbacher, D. A., \& Bissell, B. A. 1996, AJ, 111, 1880

Elmegreen, D. M., Kaufman, M., Brinks, E., Elmegreen, B. G., \& Sundin,

M. 1995a, ApJ, 453, 100 (Paper I)
Elmegreen, D. M., Sundin, M., Elmegreen, B. G., \& Sundelius, B. 1991 A\&A, 244, 52

Freeman, K. C. 1987, ARA\&A, 25, 603

Freedman, W., et al. 1994, Nature, 371, 757

Hibbard, J. E. 1995, Ph.D. thesis, Columbia University

Howard, S., Keel, W. C., Byrd, G., \& Burkey, J. 1993, ApJ, 417, 502

Irwin, J. A. 1994, ApJ, 429, 618

Kamphuis, J. J. 1993, Ph.D. thesis, University of Groningen

Klaric, M. 1993, Ph.D. thesis, University of Alabama

Noguchi, 1987, MNRAS, 228, 635

Persic, M., \& Salucci, P. 1991, ApJ, 368, 60

Sandage, A. R. 1961, The Hubble Atlas of Galaxies (Carnegie Institute of Washington, Washington)

Smith, B. J. 1994, AJ, 107, 1695

Smith, B. J. 1997a, AJ (in press)

Smith, B. J. 1997b, private communication

Smith, B. J., Struck, C., \& Pogge, R. W. 1997, ApJ, 483, 754

Strong, A. W., et al. 1988, A\&A, 207, 1

Sundin, M. 1993, Ph.D. thesis, University of Göteborg and Chalmers University of Technology, Göteborg, Sweden

Van der Hulst, J. M., Kennicutt, R. C., Crane, P. C., \& Rots, A. H. 1988, A\&A, 195, 38

Van der Kruit, P. C. 1989, in The Milky Way As a Galaxy, SAAS FEE Lecture Notes 19, edited by R. Buser and I. R. King (University Science Books, Mill Valley, CA), p. 185

Van der Kruit, P. C., \& Shostak, G. S. 1984, A\&A, 134, 258

Wray, J. D. 1988, The Color Atlas of Galaxies (Cambridge University Press, Cambridge) 


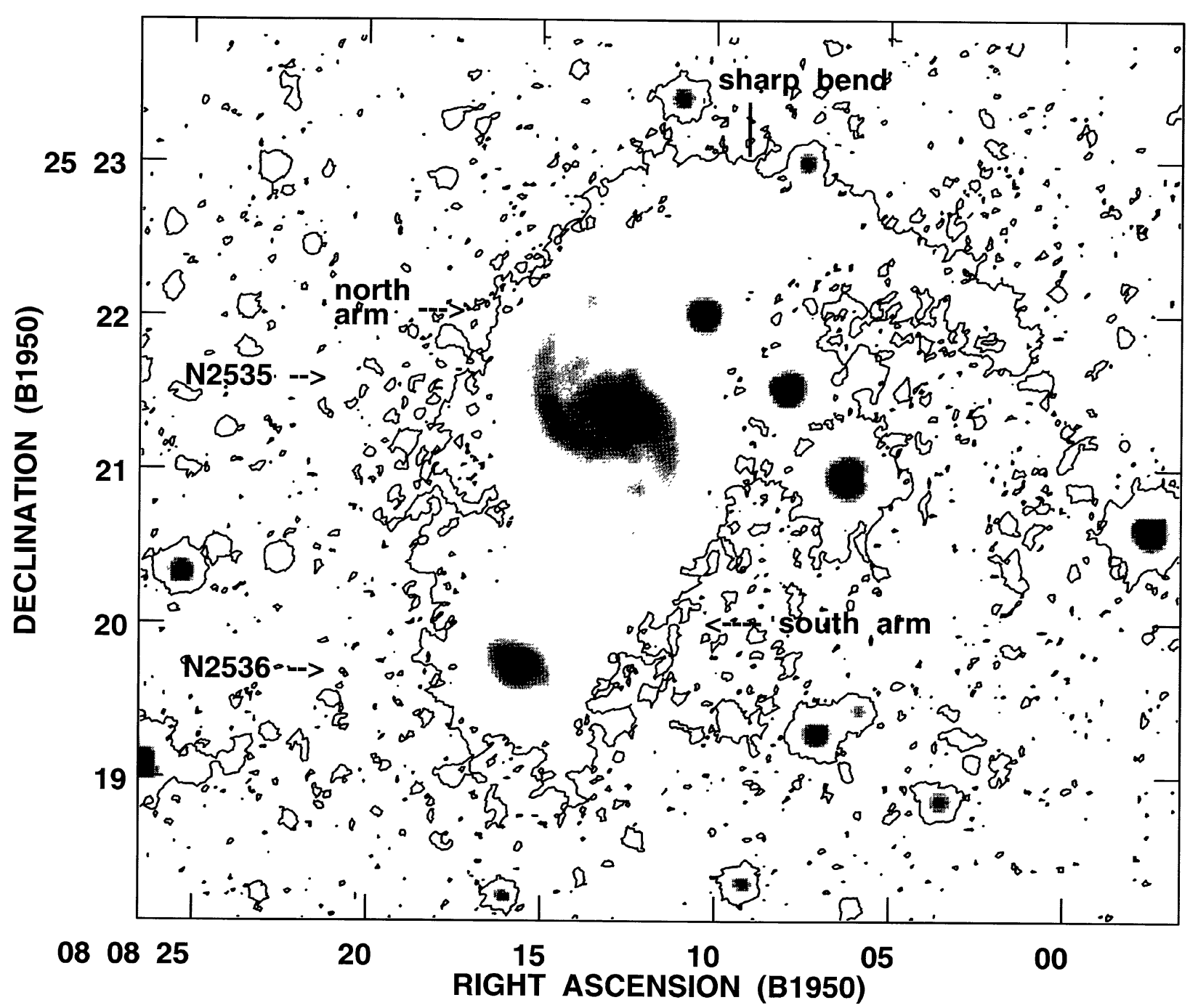

FIG. 1. $B$-band image in greyscale of NGC 2535/36 with a contour at our limiting $B$-band surface brightness (estimated as $26.5-27.0 \mathrm{mag}^{2}$ arcsec ${ }^{-2}$ ). This contour traces emission from the extended stellar envelope of NGC 2535.

Kaufman et al. (see page 2324) 
PLATE 102
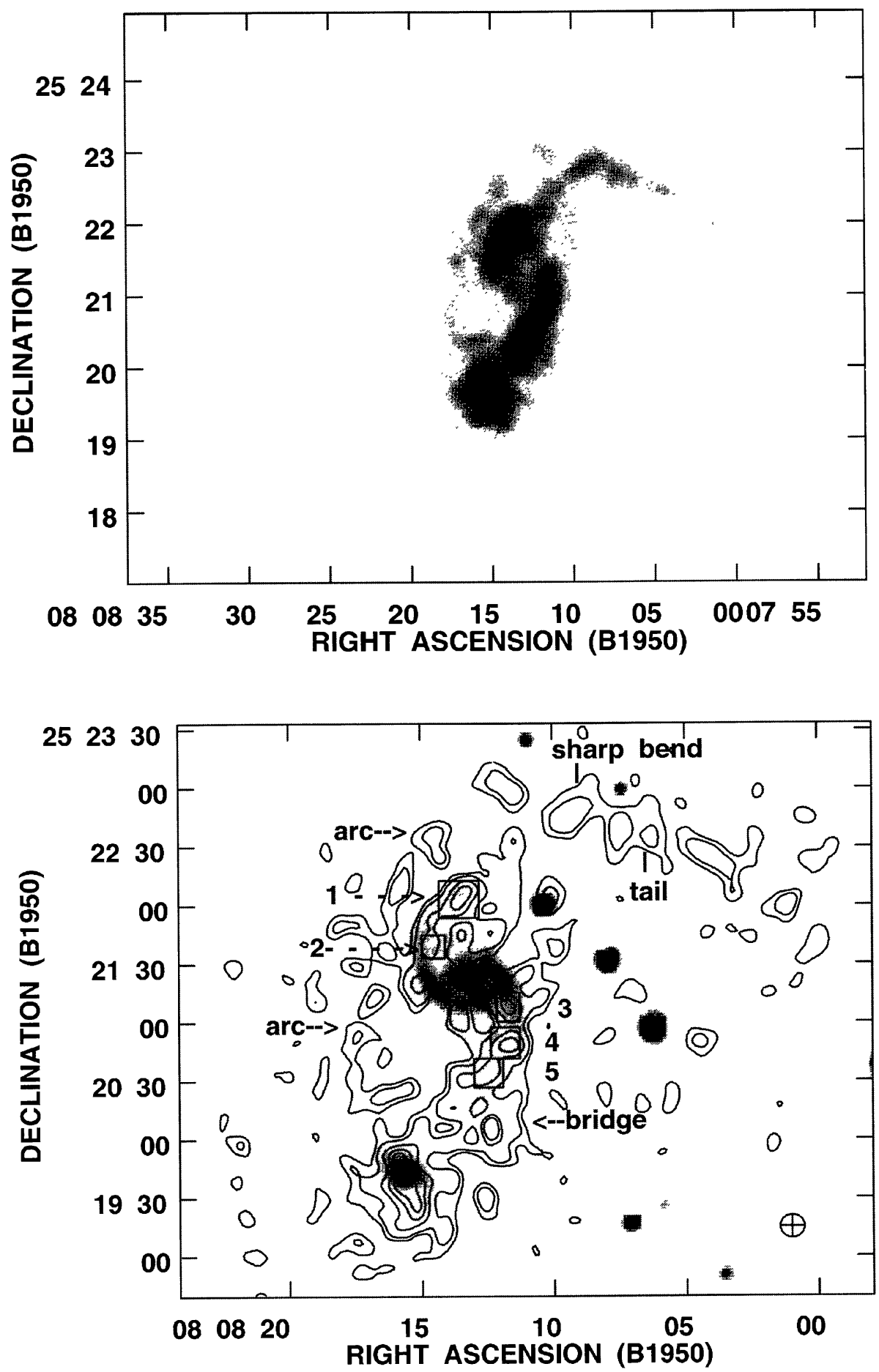

FIG. 6. Top: Greyscale display of $N(\mathrm{H}$ I $)$ for the two galaxies combined from the low resolution data (resolution $=16.7^{\prime \prime} \times 16.0^{\prime \prime}$ ). Notice the extended $\mathrm{H}$ I envelope of NGC 2535 and the elliptically-shaped arc that parallels, in projection, part of the tidal tail of NGC 2535. Bottom: Greyscale display of the $B$-band image overlaid with $N\left(\mathrm{H}\right.$ I) contours from the high resolution data. The beam symbol represents the $\mathrm{H}$ I resolution $\left(12.4^{\prime \prime} \times 11.7^{\prime \prime}\right)$. The contour levels are the same as in the bottom panel of Fig. 5. The label "arc" marks the H I elliptical arc, and the labels "tail" and "bridge" refer to the tidal tail and the tidal bridge, respectively. Numbered boxes are drawn around five massive $\mathrm{H}$ i clouds, each with an $\mathrm{H}$ I mass in excess of $10^{8}$. H $_{\odot}$. These clouds do not coincide with the most luminous blue knots in NGC 2535.

Kaufman et al. (see page 2330) 